\title{
Ioxxonony ond Systemontc \\ Survey of the Heteroptera (Hemiptera) on pastures from Espírito Santo state, Brazil: new records, range extension, and notes of potential pests
}

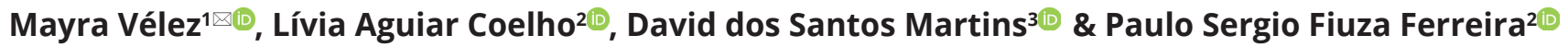

\begin{abstract}
1. Universidad Técnica Estatal de Quevedo, Facultad de Ciencias Agrarias, Quevedo, Ecuador. 2. Universidade Federal de Viçosa, Departamento de Entomologia, Viçosa, 36570-900, MG, Brazil. 3. Instituto Capixaba de Pesquisa, Assistência Técnica e Extensão Rural - INCAPER, Vitória, ES, Brazil.
\end{abstract}

\section{EntomoBrasilis 13: e907 (2020)}

\section{Edited by:}

William Costa Rodrigues

\section{Article History:}

Received: $20 . i v .2020$

Accepted: 03.viii.2019

Published: 28.viii.2020

\section{Corresponding author:}

Mayra Vélez

乃 mayracarolinavelez@gmail.com

\section{Funding agencies:}

* Conselho Nacional de Desenvolvimento Científico e Tecnológico (CNPq); Coordenação de Aperfeiçoamento de Pessoal de Nível Superior (Capes); Instituto Capixaba de Pesquisa Assistência Técnica e Extensão Rural (INCAPER)

\begin{abstract}
Espírito Santo is a Brazilian state that has a wide variety of habitats, providing a high biological diversity of fauna and flora. However, few reports had been shown about the insect diversity of this region, especially of heteropterans insects that inhabit on pasture ecosystems. In the present study, forty-three species of Heteroptera belonging to thirteen families were collected on pastures on Espírito Santo state, Brazil: Alydidae (1 sp.), Berytidae (3 sp.), Blissidae (1sp.), Coreidae (7 sp.), Miridae (16 sp.), Nabidae (1sp.), Pentatomidae (3 sp.), Pyrrhocoridae (1 sp.), Reduviidae (6 sp.), Rhopalidae (1 sp.), Rhyparochromidae (1 sp.), Thaumastocoridae (1 sp.) and Tingidae (1sp). Among the species; Cebrenis tenebrosa (Brailovsky, 1995) is recorded for the first time in Brazil. The range extension is also included for several heteropterans collected on the Espírito Santo state. The following species: Blissus aff. antillus (Leonard, 1968); Collaria oleosa (Distant, 1883); Oebalus ypsilongriseus (De Geer, 1773), and Trigonotylus tenuis (Reuter, 1893) were observed causing damages on pastures, therefore, in this study, we suggested those species as potential pastures pests. This study aims to increase the knowledge about the heteropterans and to show the presence of some pest species that may cause serious damage to pastures and consequently affect the economy of cattle breeders on the state.
\end{abstract}

Keywords: Biogeographic extension; pastureland; pests; Poaceae; true bugs.

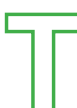
The true bug (Hemiptera: Heteroptera) is considered the largest group of insects with incomplete metamorphosis (Schuh \& Slater 1995; Weirauch \& Schuh 2011; Panizzi \& Grazia 2015). Heteroptera suborder includes such common insects as the assassin bugs, stink bugs, plant bugs, lace bugs, water striders, bedbugs, and so forth (GRAZIA \& FERNANDES 2012; PANIzZI \& GrazIA 2015). The heteropteran diversity (over 42,300 species) is distributed in approximately 75 families (Schuh \& Slater 1995; Henry 2009; Weirauch \& Schuh 2011; Grazia \& Fernandes 2012; Gullan \& CRANSTON 2014). The largest family is Miridae, with more than 11,139 species or about $25 \%$ of the true bugs (Henry 2009; Schun 2013; Ferreira et al. 2015).

Heteropterans have been studied on both basic and applied aspects worldwide (PANIzZI \& GrazIA 2015) because no other major group of insects successfully utilizes such an enormous array of different habitats (terrestrial, aquatic and semiaquatic) and feeding habits (phytophagous, predatory or hematophagous). It has been inevitable that some of them would become pests of crops. Also, there are some predaceous that would feed on those and could be potential agents of biological control. As a consequence, the interest in heteropteran has been growing especially because many species have become the principal concern in economic and medical fields (SCHAEFER \& PANIZZI 2000).

Among the cultivated plants, Poaceae family is the particular interest of humans. The economic importance of this group of plants is unquestionable, most people on earth rely on grasses, including rice, wheat, and maize, for a major portion of their diet; even to the animals like beef cattle which is raised on diets partly or wholly of grasses (KeLLoG 2001; EucLides et al. 2010).

Espírito Santo is a Brazilian state that has a wide variety of habitats, providing high biological diversity and resources for various productive activities, both land, and sea (LANI et al. 2008). About $4 \%$ of its territory is covered by natural pastures (approximately $120,000 \mathrm{ha}$ ) and around $43 \%$ by planted pastures (approximately 1,220 million ha). However, about 75,000 ha of total pastures of Espírito Santo state are degraded (IBGE 2006).

One of the causes of pasture degradation is attributed to its incorrect management. Pest attack is also a factor that had contributed to this situation (DIAS-FILHO 2011). Insect attacks on pastures affect directly the capacity of nutrient absorption by the plant, reflecting lower production of meat and milk resulting in economic losses (ALmeIDA et al. 2000).

Although heteropterans of several genera are considered a serious pest of pastures, few studies had shown the importance of this group in Brazil. Most studies had focused on the biology and control measures to Scaptocoris carvalhoi, 
Collaria, and Blissus genera (Menezes 1990; Valerio et al. 1999; Oliveira \& Malaguido 2004; Samuels \& Coracini 2004; Nardi et al. 2007; Vivan et al. 2013). Nonetheless, Heteroptera has an enormous diversity that much remains to be studied.

Espírito Santo state has shown a small number of records and descriptions of Heteroptera species in the pastures and the studies of these species are extremely scarce, elsewhere, among the factors that control pasture productivity may include the understanding of heteropterans complex. To improve the knowledge of the diversity of species of heteropterans in the pastures of the state of Espírito Santo in Brazil, the authors reported new records of species for the state and country. The distributions for the species on the state and suggestions about potential pasture pests were given.

\section{MATERIAL AND METHODS}

Heteroptera specimens were collected from pastures of twenty municipalities of Espírito Santo state, Brazil (1, Montanha; 2, Pinheiros; 3, Ecoporanga; 4, Barra de São Francisco; 5, Vila Pavão, 6, Linhares; 7, Aracruz; 8, João Neiva; 9, Colatina; 10, Baixo Guandú; 11, Itaguaçu; 12, Santa Teresa; 13. Afonso Cláudio; 14, Conceição do Castelo; 15, Venda Nova do Imigrante; 16, Castelo; 17, Ibitirama; 18, Jerónimo Monteiro; 19, Atílio Vivacqua; 20, São José do Calçado). Four sites in each municipality were sampled, comprising in a total of eighty sampled sites. The sampling sites were selected taking into account the climatic conditions of Espírito Santo which were defined in seven homogeneus climatic regions by Instituto Capixaba de Pesquisa, Assistência Técnica e Extensão Rural (INCAPER) (Figure 1).

Togenerate the map with each sampled site visited, geographic coordinates were obtained with a global positioning system (GPS) using a Garmin GPS (eTREX 10, Garmin, Brazil). All coordinates indicated on the labels of specimens examined are South (latitude) and West (longitude), respectively. The map showing the sampling sites was made with the software ArcMap (version 10.0) (ESRI 2010).

Specimens were collected using beating nets and deposited in plastic bags with pieces of paper and cotton soaked with ethyl acetate, then specimens were mounted in the lab, and examined under a Leica MZ8 stereomicroscope. Whenever required to identify, the genital capsule of males was dissected and cleared with a potassium hydroxide solution for observation. Dissected genitalia was preserved in microvials with glycerin.

All the localities are organized in north-to-south order. Abbreviations used for Brazilian states are as follows: AC, Acre; AL, Alagoas; AM, Amazonas; BA, Bahía; CE, Ceará; DF, Distrito Federal; ES, Espírito Santo; GO, Goiás, MA, Maranhão; MT, Mato Grosso; MS, Mato Grosso do Sul, MG, Minas Gerais; PA, Pará, PB, Paraíba; PR, Paraná; PE, Pernambuco, RJ, Rio de Janeiro; RN, Rio Grande do Norte; RS, Rio Grande do Sul; RO, Rondônia; SC, Santa Catarina; SP, São Paulo.

All the material examined, including sex and the complete data from the labels, is presented. New records for Espírito Santo state and to the country are indicated with an asterisk $\left.{ }^{*}\right)$. When the observations pointed to a particular taxon, but it wasn't identified any character to separate it from others that are very similar, the abbreviation aff., from latin affinis, is used to indicate affinity. In the remarks section, it is shown the Poaceae host plant in which heteropterans specimens were collected. Some pastures in which heteropterans were collected had infested by weeds or crop residuals in the outskirts, the weeds were not identified by specie. However, it was described in the manuscript in general terms.

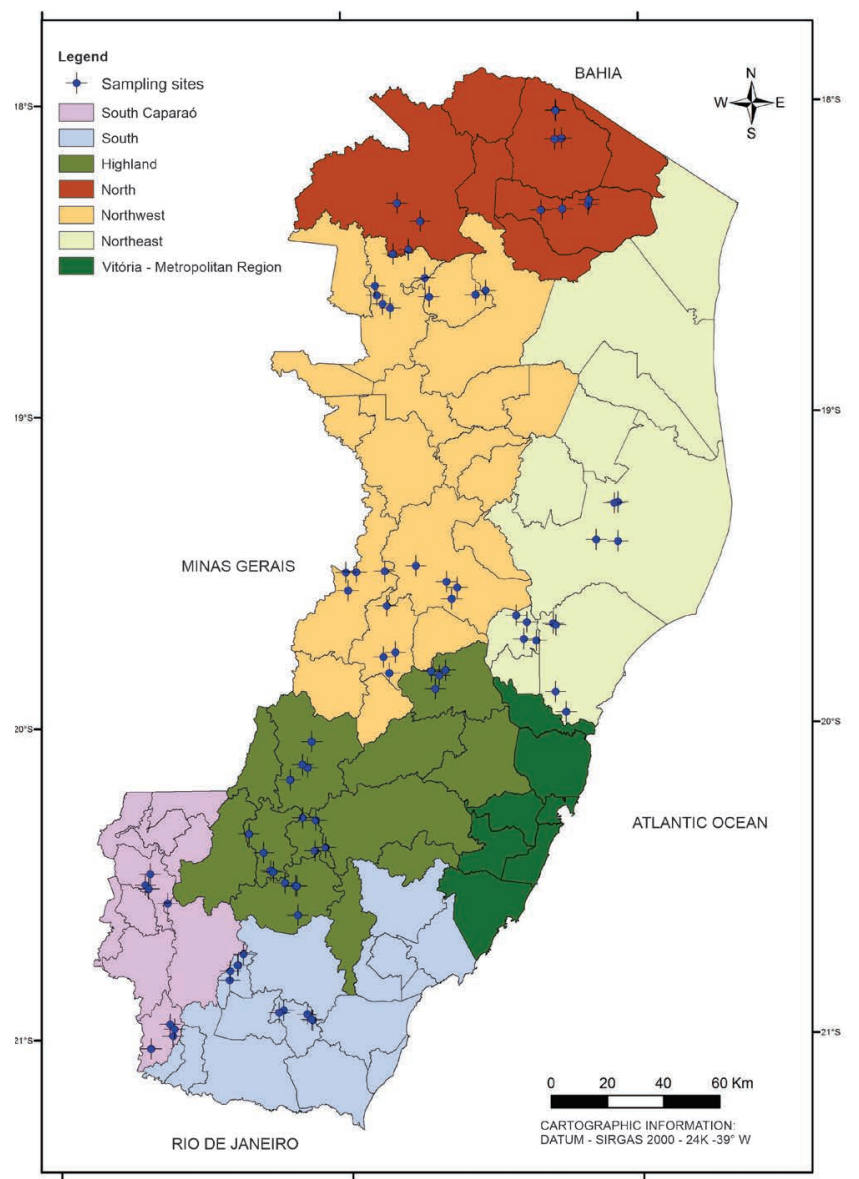

Figure 1. Map of Espírito Santo state showing the sampling sites of pastures in which heteropterans were collected.

\section{RESULTS}

We list all taxa, determined to specie level, belonging to thirteen families of Heteroptera. The families studied were Alydidae, Berytidae, Blissidae, Coreidae, Miridae, Nabidae, Pentatomidae, Pyrrhocoridae, Reduviidae, Rhopalidae, Rhyparochromidae, Thaumastocoridae, and Tingidae.

\section{Alydidae}

\section{Neomegalotomus parvus (Westwood, 1842)}

Distribution: USA, Mexico, Ecuador, Argentina, Brazil: MG, RJ, SP, RS, ES* (Jerônimo Monteiro, Ibitirama, Atílio Vivacqua, Castelo, Conceição do Castelo, Santa Teresa, Aracruz, João Neiva, Pinheiros, Vila Pavão, Ecoporanga, Barra de São Francisco) (Silva et al. 1968; PanizzI et al. 2000a; Henry \& WiLson 2004; Schaefer \& Ahmad 2008; Panizzi \& Schaefer 2015; Martins et al. 2016).

Material examined: (UFVB) 1 , Brasil - ES - Jerônimo Monteiro, Coord. 20 44'4.61", 41 ${ }^{\circ} 21^{\prime} 57.90^{\prime \prime}$, Rede devarredura, 25.II.2013, Col. M.C. Vélez. 1 §̊, Brasil - ES - Ibitirama, Ribeirão Santa Marta, Coord. 20³0'26.90", 4141'52.75", 26.II.2013, Col. M.C. Vélez. 1 j̃, 1 , Brasil - ES - Atílio Vivacqua, Retiro da lagoinha, Coord. 2055'24.25", 41¹4'47.72", 26.II.2013, Col. M.C. Vélez. 1 đ̃, Brasil - ES - Castelo, Minerva, Coord. 20³0'57.44", 4111'9.16", 02.III.2013, Col. M.C. Vélez. 1 Brasil - ES - Conc. Do Castelo, Sítio Montevideo, Coord. 20²8'17.04", 41¹5'37.22", 06.III.2013, Col. M.C. Vélez. 1 q, Brasil - ES - Santa Teresa, Sítio Natal, Coord. 1953'19.03", 4042'1.59", 08.III.2013, Col. M.C. Vélez. 1 \%, Brasil - ES Aracruz, Ribeirão do Sapí, Coord. 1940'53.97", 40¹7'55.36", 05.III.2013, Col. M.C. Vélez. 1 đ̃, Brasil - ES - João Neiva, Três Ríos, Coord. 19³9'15.81", 40²5'25.36", 13.III.2013, Col. M.C. Vélez. 1 d̃, Brasil - ES - Pinheiros, Fazenda Bom 
Gosto, Coord. 18²0'1.97", 40¹0'14.11", 19.III.2013, Col. M.C. Vélez. 1 ก., Brasil - ES - Vila Pavão, Sítio três irmãos, Coord. 18³6'32.04", 40³1'4.22", 20.III.2013, Col. M.C. Vélez. 1 ô, Brasil - ES - Ecoporanga, Sítio Huviner, Coord. 18²8'34.31", 4046’38.27, 21.III.2013, Col. M.C. Vélez. 1 ô, Brasil - ES - B. de São Francisco, Sítio dois corações, Coord. 18³7'17.99", 4053'8.53", 22.III.2013, Col. M.C. Vélez.

Remarks: Specimens of $N$. parvus were collected in pastures of Brachiaria mutica, $B$. decumbens, $B$. ruziziensis $\times B$. brizantha cv. Marandu, B. humidicola, Cynodon spp. and Panicum maximum cv. Mombaza. Pastures were associated with weeds.

\section{Berytidae}

Gampsocoris nexus (Harris, 1943)

Distribution: Peru, Bolivia, Brazil: AM, BA, MG, RJ, SP, ES* (Baixo Guandu, Montanha) (Henry 1997; Henry \& Froeschner 1998).

Material examined: (UFVB) $2 \hat{\sigma}, 2$ q, Brasil - ES - Baixo Guandu, Granja Capixaba, Coord. 19³0'39.94", 4057'54.75", 14.III.2013, Col. M.C. Vélez. 1 đ̆, Brasil - ES - Montanha, Sítio Estrela, Coord. 18'1'50.56", 40¹6'43.45", 14.III.2013, Col. M.C. Vélez.

Remarks: Specimens of G. nexus were collected in pastures of Brachiaria mutica and B. brizantha.

\section{Jalysus macer (Stål, 1859)}

Distribution: USA, Mexico, Cuba, Jamaica, Guatemala, Honduras, El Salvador, Nicaragua, Costa Rica, Panama, Colombia, Venezuela, Guyana, Ecuador, Peru, Paraguay, Argentina, Brazil: ES* (Pinheiros) (HenRY 1997; HenRY \& Froeschner 1998; Dellapé et al. 2010; Rengifo-Correa \& GonzÁlez 2011).

Material examined: (UFVB) $1 \hat{0}$, Brasil - ES - Pinheiros, Fazenda Bom Gosto, Coord. 18²0'1.97", 40¹0'14.11", 19.III.2013, Col. M.C. Vélez.

Remarks: The specimen of J. macer was collected in pastures of Brachiaria brizantha.

\section{Jalysus sobrinus (Stål, 1862)}

Distribution: Mexico, Jamaica, Guatemala, Honduras, El Salvador, Costa Rica, Panama, Nicaragua, Colombia, Venezuela, Trinidad, Ecuador, Perú, Bolivia, Paraguay, Argentina, Uruguay, Brazil: MG, SP, ES* (Afonso Cláudio) (SILVA et al. 1968; Froeschner 1981; Henry \& Froeschner 1998; Paula \& FERREIRA 2000).

Material examined: (UFVB) 1 , Brasil - ES - Afonso Cláudio, Fazenda Sermarv. Coord. 208'16.10", 41ㅇ'19.19", 05.III.2013, Col. M.C. Vélez.

Remarks: The specimen of J. sobrinus was collected in pastures of Brachiaria ruziziensis.

\section{Blissidae}

\section{Blissus aff. antillus (Leonard 1968)}

Distribution: Puerto Rico, Cuba, Cayman Islans; Dominican Republic, Grenada, Jamaica, Trinidad and Tobago, Brazil: RJ, MS, ES* Jerônimo Monteiro, Afonso Cláudio, Conceição do Castelo, Itaguaçu, Santa Teresa, Linhares, Baixo Guandu, Montanha, Ecoporanga, Barra de São Francisco, Pinheiros) (Valério et al. 1999; Samuels et al. 2002; Dellapé \& Henry 2020).
Material examined: (UFVB) 1 ก, 3 9, Brasil - ES -Jerônimo Monteiro, Sítio Boa Sorte, Coord. 2047'14.37", 41²4'43.08", 25.II.2013, Col. M.C. Vélez. 1 , Brasil - ES - Afonso Cláudio,

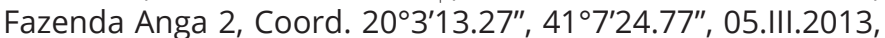
Col. M.C. Vélez. 2 j, 6 ㅇ, Brasil - ES - Itaguaçu, Sítio Bela Vista, Coord. 19³7'14.03", 4051'45.12", 07.III.2013, Col. M.C. Vélez. 1 , Brasil - ES - Santa Teresa, Sítio Natal, Coord. 1953'19.03", 4042'1.59", 08.III.2013, Col. M.C. Vélez. 1 Jे, 2 q, Brasil - ES - Baixo Guandu, Granja Capixaba, Coord. 19³0'39.94", 4057'54.75", 14.III.2013, Col. M.C. Vélez. 1 กิ, Brasil - ES - Baixo Guandu, Fazenda São Sebastião, Coord. 19³0'32.76",4052'7.53", 14.III.2013, Col. M.C. Vélez. 1 ㅇ, Brasil - ES - Ecoporanga, Santa Teresinha, Coord. 18²9'20.89", 4049'45.38", 21.III.2013, Col. M.C. Vélez. 1 đ, Brasil - ES - B. de São Francisco, Sítio dois corações, Coord. 18³7'17.99", 4053'8.53", 22.III.2013, Col. M.C. Vélez. (USNM) 1 specimen, Brasil - ES - Conc. Do Castelo, Sítio Monforte

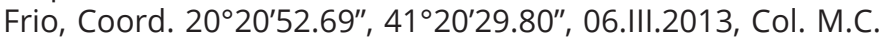
Vélez. 1 specimen, Brasil - ES - Linhares, Fazenda Graciosa II, Coord. 19¹7'43.17", 405'18.90", 11.III.2013, Col. M.C. Vélez. 2 ㅇ, Brasil - ES - Montanha, Sítio Estrela, Coord. 18¹'50.56", 40¹6'43.45", 14.III.2013, Col. M.C. Vélez. 1 + , Brasil - ES - Baixo Guandu, Granja Capixaba, Coord. 19³0'39.94", 4057'54.75", 14.III.2013, Col. M.C. Vélez.

Remarks: Specimens of $B$. antillus were collected in pastures of Brachiaria mutica, $B$. brizantha, $B$. decumbens, $B$. mutica $\times B$. arrecta, Cynodon spp. and Panicum maximum cv. Mombaza.

\section{Coreidae}

\section{Cebrenis duplicata (Brailovsky, 1995)}

Distribution: Peru, Brazil: RJ, SC, ES* (João Neiva) (BrallovskY 1995; CRUCEs \& Vergara 2015).

Material examined: (UNAM) 2 specimens, Brasil - ES - João Neiva, Sítio Boa Amizade, Coord. 1940'36.77", 40²3'16.08", 13.III.2013, Col. M.C. Vélez.

Remarks: Specimens of C. duplicata were collected in pastures of Brachiaria brizantha. Pastures were associated with weeds.

\section{Cebrenis tenebrosa (Brailovsky 1995)}

Distribution for C. tenebrosa (Brailovsky, 1995): Colombia, Venezuela, Brazil*: ES* (Pinheiros) (Brallovsky 1995).

Material examined: (UNAM) 1 specimen, Brasil - ES - Pinheiros, Fazenda Bom Gosto, Coord. 18²0'1.97", 40¹0'14.11", 19.III.2013, Col. M.C. Vélez.

Remarks: Specimens of $C$. tenebrosa were collected in pastures of Brachiaria brizantha. Pastures were associated with weeds.

\section{Hypselonotus interruptus (Hahn, 1833)}

Distribution: Mexico, Guatemala, Belize, Honduras, El Salvador, Costa Rica, Panama, Colombia, Venezuela, Trinidad and Tobago, Ecuador, Peru, Bolivia, Paraguay, Argentina, Brazil: PA, PE, MG, SP, RS, ES (Linhares, Jerônimo Monteiro, Santa Teresa) (BLöte 1938; Silva et al. 1968; Thum \& Costa 1997; Dellapé P. et al. 2015; Martins et al. 2016).

Material examined: (UFVB) 1 , Brasil - ES - Jerônimo Monteiro, Sítio Boa Sorte, Coord. 2047'14.37", 41²4'43.08", 25.II.2013, Col. M.C. Vélez. 1 + , Brasil - ES - Santa Teresa, Sítio Natal, Coord. 1953'19.03", 4042'1.59", 08.III.2013, Col. M.C. Vélez.

Remarks: Specimens of $H$. interruptus were collected in pastures of Brachiaria brizantha and B. mutica $\times$ B. arrecta. 
Pastures were associated with weeds.

\section{Merocoris elevatus (Spinola, 1837)}

Distribution: Bolivia, Argentina, Brazil: DF, ES* (Atílio Vivacqua, São José do Calçado) (StÅl 1870; Kormilev 1954).

Material examined: (UFVB) $1 \hat{\jmath}$, Brasil - ES - Atílio Vivacqua, Fazenda Reta, Coord. 2054'53.84", 4113'49.75", 27.Il.2013, Col. M.C. Vélez. 1 +, Brasil - ES - São José do Calçado, Fazenda Soledade, Coord. 2057'19.19", 41³7'16.34", 28.Il.2013, Col. M.C. Vélez.

Remarks: Specimens of $M$. elevatus were collected in pastures of Brachiaria brizantha and $B$. decumbens. Pastures were associated with weeds.

\section{Phthiacnemia picta (Drury, 1773)}

Distribution: Mexico, Cuba, Honduras, El Salvador, Nicaragua, Costa Rica, Puerto Rico, Saint Martin, Colombia, Venezuela, Suriname, Guyana Paraguay, Argentina, Uruguay, Brazil: PE, AL, MG, RJ, SP, SC, ES (Linhares, João Neiva) (Costa LIMA 1940; Silva et al. 1968; Brailovsky 2009; Dellapé P. et al. 2015; Martins et al. 2016).

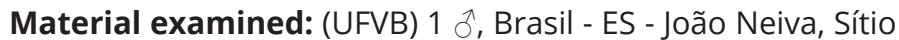
Boa Amizade, Coord. 1940'36.77", 40²3'16.08", 13.III.2013, Col. M.C. Vélez.

Remarks: The specimen of $P$. picta was collected on pasture of Brachiaria brizantha. Pastures were associated with weeds.

\section{Vilga sanctipauli (Dolling, 1977)}

Distribution: Argentina, Brazil: SP, ES* (Jerônimo Monteiro, Ibitirama, Montanha) (DoluIng 1977; Olivera et al. 2017).

Material examined: (UNAM) 2 specimens, Brasil - ES Jerônimo Monteiro, Coord. 2049'1.31", 41²4'53.04", Rede de varredura, 25.II.2013, Col. M.C. Vélez. 2 specimens, Brasil - ES - Jerônimo Monteiro, Fazenta Luzitania, Coord. 2046'10.64", 4123'4.32", 25.Il.2013, Col. M.C. Vélez. 1 specimen, Brasil - ES - Ibitirama, Córrego da lagoa, Coord. 20²8'20.61", 4140'50.80", 26.II.2013, Col. M.C. Vélez. 1 specimen, Brasil - ES - Montanha, Fazenda Cinelândia, Coord. 187'16.91", 40¹5'32.49", 18.III.2013, Col. M.C. Vélez.

Remarks: Specimens of $V$. sanctipauli were collected on pastures of Brachiaria brizantha and Panicum maximum cV. Mombaza. Pastures were associated with weeds.

\section{Vilga westwoodi (Kolenati, 1845)}

Distribution: Panama, Colombia, Trinidad, Guyana, Bolivia, Paraguay, Argentina, Brazil: AM, PA, MT, BA, ES* Jerônimo Monteiro) (DOLLING 1977; Forero 2006).

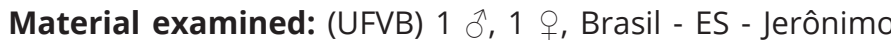
Monteiro, Fazenta Luzitania, Coord. 2046'10.64", 41 ${ }^{\circ} 23^{\prime} 4.32^{\prime \prime}$, 25.II.2013, Col. M.C. Vélez.

Remarks: Specimens of $V$. westwoodi were collected on pastures of Panicum maximum cv. Mombaza. Pastures were associated with weeds.

\section{Miridae}

\section{Campyloneuropsis infumatus (Carvalho, 1947)}

Distribution: Peru, Argentina, Brazil: MG, SC, ES* (Ibitirama) (Carvalho 1947; Schuh 2013; Carpintero et al. 2014).

Material examined: (UFVB) $1 \hat{\jmath}$, Brasil - ES - Ibitirama, Ribeirão Santa Marta, Coord. 20³0'26.90", 4141'52.75",
26.II.2013, Col. M.C. Vélez.

Remarks: The specimen of $C$. infumatus was collected on pastures of Brachiaria decumbens.

\section{Collaria oleosa (Distant, 1883)}

Distribution: Mexico, Cuba, Puerto Rico, Guatemala, El Salvador, Costa Rica, Panama, Colombia, Ecuador, Peru, Brazil: AM, PA, AC, RO, MT, BA, GO, MG, RJ, SP, PR, SC, ES (Linhares, Ibitirama, São José do Calçado, Venda Nova do Imigrante, Afonso Cláudio, Conceição do Castelo, Itaguaçu, Santa Teresa, Aracruz, Água Doce do Norte, Baixo Gandu, Colatina) (CARValho \& Fontes 1981; Silva et al.1994; HeRnÁndez \& Henry 2010; Schuh 2013; Martins et al. 2016; Morales et al. 2016).

Material examined: (UFVB) 1 specimen, Brasil - ES - Ibitirama, Córrego da lagoa, Coord. 20²8'20.61", 4140'50.80", 26.II.2013, Col. M.C. Vélez. 1 specimen, Brasil - ES - São José

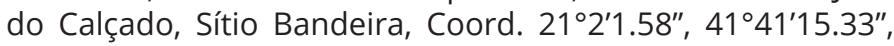
28.II.2013, Col. M.C. Vélez. 3 đ, 3 q, Brasil - ES - Venda Nova, Sítio São José, Coord. 20²4'19.59", 41 7'1.47", 04.III.2013, Col. M.C. Vélez. 1 స, 3 q, Brasil - ES - Afonso Cláudio, Sítio Bom Sucesso Coord. 20¹0'32.29", 41¹1'53.65", 05.III.2013, Col. M.C. Vélez. 2 A, Brasil - ES - Conc. Do Castelo, Sítio Monforte Quente, Coord. 20²4'31.20", 41'17'30.96", 06.III.2013, Col. M.C. Vélez. 1 specimen, Brasil - ES - Itaguaçu, Sítio Ninke, Coord. 1947'3.50", 4052'32.96", 07.III.2013, Col. M.C. Vélez. 1 specimen, Brasil - ES - Santa Teresa, Sítio Natal, Coord. 1953'19.03", 4042'1.59", 08.III.2013, Col. M.C. Vélez. 1 ', Brasil - ES - Aracruz, Ribeirão do Sapí, Coord. 1940'53.97", 40¹7'55.36", 05.III.2013, Col. M.C. Vélez.

Remarks: Specimens of $C$. oleosa were collected on pastures of Brachiaria mutica, B. brizantha, B. decumbens, B. ruziziensis, Cynodon spp., Panicum maximum cv. Mombaza, P. maximum CV. Tanzania.

\section{Cyrtocapsus femoralis (Reuter, 1892)}

Distribution: Venezuela, French Guiana, Brazil: MG, RS, ES* (Jerônimo Monteiro) (Barcellos et al. 2011; Ferreira \& Henry 2011; SснUн 2013).

Material examined: (UFVB) 6 ก, 4 , Brasil - ES - Jerônimo Monteiro, Fazenta Luzitania, Coord. 20 $46^{\prime} 10.64^{\prime \prime}, 41^{\circ} 23^{\prime} 4.32^{\prime \prime}$, 25.II.2013, Col. M.C. Vélez.

Remarks: Specimens of C. femoralis were collected on pastures of Brachiaria brizantha and Panicum maximum cV. Mombaza.

\section{Dolichomiris linearis (Reuter, 1882)}

Distrubution: India, Ghana, Canary Island, New Hebrides, Fiji, Australia, New Guinea, New Caledonia, USA, México, Cuba, Puerto Rico, Guatemala, Honduras, El Salvador, Venezuela, Suriname, Ecuador, Peru, Brazil: MT, BA, GO, MG, RJ, SP, SC, ES* (Conceição do Castelo, Venda Nova do Imigrante, Ibitirama, Barra de São Francisco) (CARValho 1975a; Ferreira et al. 2001; Sснин 2013).

Material examined: (UFVB) 1 specimen, Brasil - ES - Conc. Do Castelo, Sítio Monforte Frio, Coord. 20²0'52.69", 41²0'29.80", 06.III.2013, Col. M.C. Vélez. 1 , Brasil - ES -Venda Nova, Pe da Serra, Coord. 20²4'19.59", 20¹8'24.13", 416'43.77", 04.III.2013, Col. M.C. Vélez. 4 j̃, 1 ㅇ, Brasil - ES - Venda Nova, Sítio São José, Coord. 20²4'19.59", 417'1.47", 04.III.2013, Col. M.C. Vélez. 1 specimen, Brasil - ES - Ibitirama, Córrego da

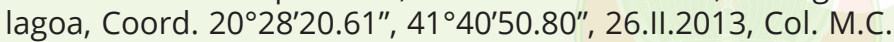
Vélez. 1 1, 1 +, Brasil - ES - B. de São Francisco, Pau de alho, Coord. 18³9'48.13", 4050'26.50", 22.III.2013, Col. M.C. Vélez.

Remarks: Specimens of $D$. linearis were collected on pastures 
of Brachiaria brizantha, B. decumbens, and Cynodon spp.

\section{Engytatus varians (Distant, 1884)}

Distribution: USA, México, Cuba, Dominican Republic, Puerto Rico, Guatemala, Nicaragua, Suriname, Ecuador, Argentina, Brazil: GO, MG, SP, ES* (Castelo) (Carvalho 1947; Ferreira \& Henry 2011; Martínez et al. 2014).

Material examined: (UFVB) 1 , Brasil - ES - Castelo, Minerva, Coord. 20³0'57.44", 41¹1'9.16", 02.III.2013, Col. M.C. Vélez.

Remarks: The specimen of $E$. varians was collected on pastures of Brachiaria decumbens. Pastures were associated with weeds.

\section{Garganus gracilentus (Stål, 1860)}

Distribution: Colombia, Venezuela, Ecuador, Peru, Paraguay, Argentina, Brazil: MG, RJ, SC, SP, MT, ES* (Ibitirama, Venda Nova do Imigrante) (Silva et al.1968; CarvalHo \& Afonso 1977; Ferreira et al. 2001; SCHUH 2013; CARPInTERo et al. 2014).

Material examined:(UFVB) 1 , Brasil - ES - Ibitirama, Córrego da passagem, Coord. 20³4'1.75", 41 ³7'21.87", 26.II.2013, Col. M.C. Vélez. 1 đ̊, Brasil - ES - Venda Nova, Fazenda Luis Uliana, Coord. 20²3'42.20", 414'49.16", 04.III.2013, Col. M.C. Vélez.

Remarks: Specimens of G. gracilentus were collected on pastures of Brachiaria brizantha and Panicum maximum cv. Mombaza.

\section{Horciasinus signoreti (Stål, 1859)}

Distribution: Colombia, Venezuela, French Guiana, Peru, Bolivia, Paraguay, Argentina, Uruguay, Brazil: AP, AM, PA, MA CE, PB, MT, BA, DF, GO, MG, RJ, SP, PR, SC, RS, ES (Linhares, Afonso Cláudio) (Carvalho \& Jurberg 1976; Ferreira et al. 2001; SCHUH 2013).

Material examined: (UFVB) $5 \hat{0}, 3$ ㅇ, Brasil - ES - Afonso Cláudio, Fazenda Sermarv, Coord. 208'16.10", 418'19.19", 05.III.2013, Col. M.C. Vélez.

Remarks: Specimens of $H$. signoreti were collected on pastures of Brachiaria ruziziensis and Cynodon spp.

\section{Macrolophus praeclarus (Distant, 1884)}

Distribution: Mexico, Cuba, Puerto Rico, Guatemala, Colombia, Venezuela, Suriname, Brazil: GO, MG, SC, ES* (São José do Calçado) (Carvalho 1945; Carvalho \& Afonso 1977; Hernández \& Henry 2010; Ferreira \& Henry 2011; Schum 2013).

Material examined: (UFVB) 1 specimen, Brasil - ES - São José do Calçado, Fazenda Vida Nova Soledade, Coord. 2058'19.81", 41³6'19.54", 28.II.2013, Col. M.C. Vélez.

Remarks: The specimen of $M$. praeclarus was collected on pastures of Panicum maximum cv Mombaza. Pastures were associated with weeds.

\section{Polymerus testaceipes (Stål, 1860)}

Distribution: USA, Mexico, Cuba, Jamaica, Puerto Rico, El Salvador, Nicaragua, Venezuela, St. Vincent, Guyana, Suriname, French Guiana, Ecuador, Peru, Paraguay, Argentina, Brazil: AM, PA, AC, PE, AL, RO, MT, BA, GO, MG, RJ, SP, PR, SC, ES (Guarapari, Linhares, Venda Nova do Imigrante, Afonso Cláudio, Ecoporanga) (CARValho 1975b; Carvalho \& Afonso 1977; Ferreira 1980, Ferreira et al. 2001; Hernández \& Henry 2010; Schuh 2013).

Material examined: (UFVB) 1 , Brasil - ES - Venda Nova, Sítio São José, Coord. 20²4'19.59", 41'1.47", 04.III.2013,
Col. M.C. Vélez. 2 đ, 1 , Brasil - ES - Afonso Cláudio, Fazenda Sermarv, Coord. 208'16.10", 41ㅇ'19.19", 05.III.2013, Col. M.C. Vélez. 1 đ̄, 4 ㅇ, Brasil - ES - Ecoporanga, Sítio do Divino, Coord. 18`19'33.39", 4048'52.69", 21.III.2013, Col. M.C. Vélez.

Remarks: Specimens of $P$. testaceipes were collected on pastures of Brachiaria brizantha, $B$. humidicola, $B$. ruziziensis, and Cynodon spp. Pastures were associated with weeds.

\section{Pycnoderes quadrimaculatus (Guerin-Meneville, 1857)}

Distribution: USA, Cuba, Dominican Republic, Puerto Rico, Guatemala, Honduras, Nicaragua, Panama, Colombia, Suriname, Peru, Brazil: MT, MG, ES* (Jerônimo Monteiro) (Carvalho \& Afonso 1977; Ferreira \& Henry 2011; Schuh 2013).

Material examined: (UFVB) 1 त̂, Brasil - ES - Jerônimo Monteiro, Coord. 2049'1.31", 41²4'53.04", Rede de varredura, 25.II.2013, Col. M.C. Vélez.

Remarks: The specimen of $P$. quadrimaculatus was collected on pastures of Brachiaria brizantha. Pastures were associated with weeds.

\section{Sericophanes ornatus (Berg, 1878)}

Distribution: Guyana, Suriname, Bolivia, Chile, Paraguay, Argentina, Brazil: MT, GO, MG, RJ, SP, SC, ES (Afonso Cláudio, Itaguaçu), (Carvalho 1944; Carvalho \& Afonso 1977; Carvalho \& Costa 1988; Ferreira et al. 2001; Schuh 2013; Carpintero et al. 2014; MARTINs et al. 2016).

Material examined: (UFVB) $1 \hat{\jmath}$, Brasil - ES - Afonso Cláudio, Fazenda Sermarv, Coord. 208'16.10", 41ㅇ'19.19", 05.III.2013, Col. M.C. Vélez. 1 ग̃, Brasil - ES - Itaguaçu, Sítio Bela Vista, Coord. 19³7'14.03", 4051'45.12", 07.III.2013, Col. M.C. Vélez.

Remarks: Specimens of S. ornatus were collected on pastures of Brachiaria mutica e Brachiaria ruziziensis.

\section{Sthenaridea carmelitana (Carvalho, 1948)}

Distribution: México, Puerto Rico, Nicarágua, Colombia, Suriname, Peru, Paraguay, Argentina, Brazil: AL, MG, RJ, SC, RS, ES* (Venda Nova do Imigrante, Afonso Cláudio, Conceição do Castelo, Santa Teresa, Ibitirama, Atílio Vivacqua) (CARVAlHo 1948; Carvalho \& Afonso 1977; Schuh \& Schwartz 1988; Ferreira et al. 2001; Barcellos et al. 2011; SCHUH 2013).

Material examined: (UFVB) 2 , Brasil - ES - Venda Nova,

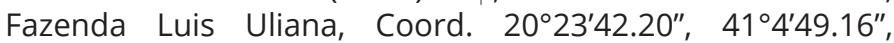
04.III.2013, Col. M.C. Vélez. 1 đ, 1 ㅇ, Brasil - ES - Venda Nova, Sítio São José, Coord. 20²4'19.59", 417'1.47", 04.III.2013, Col. M.C. Vélez. 1 specimen, Brasil - ES - Afonso Cláudio, Sítio Bom Sucesso, Coord. 20¹0'32.29", 41¹1'53.65", 05.III.2013, Col. M.C. Vélez. 1 †, Brasil - ES - Conc. Do Castelo, Sítio Montevideo, Coord. 20²8'17.04", 4115'37.22", 06.III.2013, Col. M.C. Vélez. 1 ㅇ, Brasil - ES - Santa Teresa, Sítio Natal, Coord. 1953'19.03", 4042'1.59", 08.III.2013, Col. M.C. Vélez. 1 specimen, Brasil - ES - Ibitirama, Barra de Santa Marta, Coord. 20³1'12.78", 4141'9.94", 26.II.2013, Col. M.C. Vélez. 1 specimen, Brasil - ES - Atílio Vivacqua, Independência, Coord. 2057'0.10", 417'56.99", 27.II.2013, Col. M.C. Vélez.

Remarks: Specimens of S. carmelitana were collected on pastures of Brachiaria brizantha, $B$. decumbens, $B$. ruziziensis, Cynodon spp. Panicum maximum cv. Mombaza.

\section{Taylorilygus apicalis (Fieber, 1861)}

Distribution: Spain, Switzerland, Australia, Madagascar, Turkey, Nubia, Egypt, Korea, USA, Mexico, Cuba, Puerto Rico, Guatemala, El Salvador, Panama, Colombia, Suriname, Ecuador, Peru, Chile, Paraguay, Argentina, Uruguay, Brazil: MG, 
ES* (Venda Nova do Imigrante, Afonso Cláudio, Conceição do Castelo) (Carvalho \& Afonso 1977; Ferreira et al. 2001; Hernández \& HenRy 2010; SchuH 2013; CarPINTERo et al. 2014).

Material examined: (UFVB) 1 specimen, Brasil - ES Venda Nova, Sítio São José, Coord. 20²4'19.59", 417'1.47", 04.III.2013, Col. M.C. Vélez. 1 đ̃, Brasil - ES - Afonso Cláudio, Fazenda Sermarv, Coord. 208'16.10", 41 '8'19.19", 05.III.2013, Col. M.C. Vélez. 1 ô, 1 q, Brasil - ES - Conc. Do Castelo, Sítio Montevideo, Coord. 20²8'17.04", 41ํ15'37.22", 06.III.2013, Col. M.C. Vélez.

Remarks: Specimens of T. apicalis were collected on pastures of Brachiaria brizantha, Panicum maximum cv. Mombaza. Pastures were associated with weeds.

\section{Trigonotylus tenuis (Reuter, 1893)}

Distribution: New Guinea, New Britain, USA, Mexico, Cuba, Puerto Rico, Guatemala, El Salvador, Costa Rica, Venezuela, Peru, Argentina, Brazil: BA, MG, ES* (Venda Nova do Imigrante, Afonso Cláudio, Colatina, Baixo Guandu) (CARvalho 1975a; Ferreira et al. 2001; Vivas et al. 2005; SchuH 2013; Carpintero et al. 2014).

Material examined: (UFVB) 3 3,3 , Brasil - ES - Venda Nova, Sítio São José, Coord. 20²4'19.59", 417'1.47", 04.III.2013, Col. M.C. Vélez. 2 đ̃, 5 , Brasil - ES - Afonso Cláudio, Fazenda

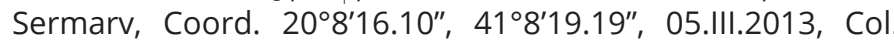
M.C. Vélez. 1 †, Brasil - ES - Afonso Cláudio, Sítio Bom Sucesso, Coord. 20¹0'32.29", 41¹1'53.65", 05.III.2013, Col. M.C. Vélez. 1 , Brasil - ES - Colatina, Sítio das Palmeiras, Coord. 19³5'57.95", 40³8'30.77", 14.III.2013, Col. M.C. Vélez. 1 specimen, Brasil - ES - Baixo Guandu, Holz, Coord. 19³0'40.08", 41ํ⒈54", 14.III.2013, Col. M.C. Vélez.

Remarks: Specimens of $T$. tenuis were collected on pastures of Cynodon spp.

\section{Tupiocoris cucurbitaceus (Spinola, 1852)}

Distribution: Mexico, Colombia, Ecuador, Peru, Chile, Argentina, Brazil: SC, ES* (Venda Nova do Imigrante). (CARVAlHo \& Afonso 1977; Ferreira \& Henry 2011; Schum 2013; Carpintero et al. 2014).

Material examined: (UFVB) 1 , Brasil - ES - Venda Nova, Sítio São José, Coord. 20²4'19.59", 417'1.47", 04.III.2013, Col. M.C. Vélez.

Remarks: Specimens of $T$. cucurbitaceus were collected on pastures of Cynodon spp. Pastures were associated with weeds.

\section{Tytthus neotropicalis (Carvalho, 1954)}

Distribution: USA, Mexico, Cuba, Puerto Rico, Haiti, Belize, Nicaragua, Panama, Venezuela, Guyana, Suriname, Ecuador, Peru, Bolivia, Paraguay, Argentina, Brazil: AM, PA, CE, RO, MG, RJ, SP, PR, SC, ES (Linhares, Venda Nova do Imigrante, Baixo Guandu) (Ferreira et al. 2001, Hernández \& Henry 2010; Henry 2012; Schun 2013; Carpintero et al. 2014; Martins et al. 2016).

Material examined: (UFVB) 2 $\curvearrowright, 2$, Brasil - ES - Venda Nova, Sítio São José, Coord. 20²4'19.59", 417'1.47", 04.III.2013, Col. M.C. Vélez. 1 specimen, Brasil - ES - Baixo Guandu, Holz, Coord. 19³0'40.08", 41ํ'1.54", 14.III.2013, Col. M.C. Vélez.

Remarks: Specimens of $T$. neotropicalis were collected on pastures of Cynodon spp.

\section{Nabidae}

Nabis capsiformis (Germar 1837)
Distribution: USA, México, Guyana, Peru, Chile, Uruguay, Argentina, Brazil: PA, MS, RJ, ES* (Ibitirama, São José do Calçado, Castelo, Venda Nova do Imigrante, Conceição do Castelo, Itaguaçu, João Neiva, Colatina, Baixo Guandu) (OJedAPeña 1971; Cornelis \& Coscarón 2013).

Material examined: (UFVB) 1 , Brasil - ES - Ibitirama, Ribeirão Santa Marta, Coord. 20³0'26.90", 41441'52.75", 26.II.2013, Col. M.C. Vélez. $1{ }^{\circledR}$, Brasil - ES - São José do Calçado, Fazenda Soledade, Coord. 2057'19.19", 41³7'16.34", 28.Il.2013, Col. M.C. Vélez. 1 o, 1 †, Brasil - ES - Castelo, Minerva, Coord. 20³0'57.44", 41¹1'9.16", 02.III.2013, Col. M.C. Vélez. 1 , Brasil - ES - Venda Nova, Fazenda Luis Uliana, Coord. 20²3'42.20", 414'49.16", 04.III.2013, Col. M.C. Vélez. 1 ô, Brasil - ES - Conc. Do Castelo, Sítio Monforte Quente, Coord. 20²4'31.20", 41¹7'30.96", 06.III.2013, Col. M.C. Vélez. 1 đ̃, 1 +, Brasil - ES - Itaguaçu, Sítio Bela Vista, Coord. 19³7'14.03", 4051'45.12", 07.III.2013, Col. M.C. Vélez. 1 , Brasil - ES João Neiva, Fazenda Jua, Coord. 1944'9.33", 40²1'21.53", 13.III.2013, Col. M.C. Vélez. 1 q, Brasil - ES - Colatina, Sítio Briel, Coord. 19³2'39.37", 40³9'33.57", 14.III.2013, Col. M.C. Vélez. 1 ․, Brasil - ES - Baixo Guandu, Holz, Coord. 19³0'40.08", 41ํ'1.54", 14.III.2013, Col. M.C. Vélez.

Remarks: Specimens of $N$. capsiformis were collected on pastures of Brachiaria mutica, B. brizantha, B. decumbens, Cynodon spp.

\section{Pentatomidae}

\section{Dichelops melacanthus (Dallas, 1851)}

Distribution: Colombia, Venezuela, Peru, Bolivia, Paraguay, Argentina, Uruguay, Brazil: MS, PR, SC, RS, ES* Jerônimo Monteiro, Atílio Vivacqua) (Grazıa 1978; Panizzı et al. 2000b; Chocorosqui \& Panizzi 2004; Panizzl et al. 2013; Dellapé et al. 2015).

Material examined: (UFVB) 1 त, Brasil - ES - Jerônimo Monteiro, Coord. 2044'4.61", 41 $21^{\prime} 57.90^{\prime \prime}$, Rede de varredura, 25.II.2013, Col. M.C. Vélez. 1 đ̃, Brasil - ES - Atílio Vivacqua, Independência, Coord. 2057'0.10", 41 '7'56.99", 27.II.2013, Col. M.C. Vélez.

Remarks: Specimens of D. melacanthus were collected on pastures of Brachiaria brizantha.

\section{Oebalus ypsilongriseus (De Geer, 1773)}

Distribution: Colombia, Guyana, Suriname, Peru, Paraguay, Argentina, Uruguay, Brazil: PA, GO, SP, RS, ES* (São José do Calçado, Castelo, Venda Nova do Imigrante, Afonso Cláudio, Santa Teresa, Aracruz, João Neiva, Colatina, Baixo Guandu, Vila Pavão, Ecoporanga, Barra de São Francisco) (SAILER 1944; Silva et al. 1968; Panizzi et al. 2000b; Rebagliati et al. 2005; Dellapé et al. 2015).

Specimens examined: (UFVB) 1 , Brasil - ES - São José do Calçado, Sítio bom futuro, Coord. 2059'37.59", 41³6'40.75", 28.ll.2013, Col. M.C. Vélez. 2 ô, 2ㅇ, Brasil - ES - Castelo, Minerva, Coord. 20³0'57.44", 41'11'9.16", 02.III.2013, Col. M.C. Vélez. 2 đ̃, Brasil - ES - Venda Nova, Fazenda Luis Uliana, Coord. 20²3'42.20", 414'49.16", 04.III.2013, Col. M.C. Vélez. 1 , Brasil - ES - Afonso Cláudio, Fazenda Sermav, Coord.

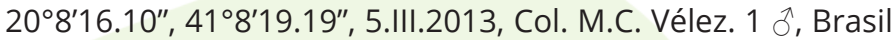
- ES - Santa Teresa, Estancia Paraiso, Coord. 1949'43.55", 40³9'56.81", 08.III.2013, Col. M.C. Vélez. 3 ô, Brasil - ES Aracruz, São José, Coord. 1941'9.49", 40¹7'19.36", 12.III.2013, Col. M.C. Vélez. 1 đ̃, Brasil - ES - João Neiva, Sítio Boa Amizade, Coord. 1940'36.77", 40²3'16.08", 13.III.2013, Col. M.C. Vélez. 2 ㅅ, 2 o, Brasil - ES - Colatina, IFES Campus Itapina, Coord. 19²9'32.00", 4045'45.67", 14.III.2013, Col. M.C. Vélez. 1 ô, Brasil - ES - Baixo Guandu, Sítio Seriema, Coord. 19³4'9.52", 
4059'38.36", 14.III.2013, Col. M.C. Vélez. 1 specimen, Brasil - ES - Vila Pavão, Sítio Boa Esperança, Coord. 18³4'0.84", 4043'24.04", 20.III.2013, Col. M.C. Vélez. 2 ô, 2 ․, Brasil - ES - Ecoporanga, Santa Teresinha, Coord. 18²9'20.89", 4049'45.38", 21.III.2013, Col. M.C. Vélez. 1 đ̃, Brasil - ES B. de São Francisco, Sítio Boa Vista, Coord. 18³5'28.79", 4053'30.28", 22.III.2013, Col. M.C. Vélez.

Remarks: Specimens of $O$. ypsilongriseus were collected on pastures of Brachiaria brizantha, B. decumbens, Cynodon spp. Panicum maximum cv. Mombaza.

\section{Piezodorus guildinii (Westwood, 1837)}

Distribution: USA, Mexico, Cuba, Jamaica, Haiti, Guatemala, Honduras, Nicaragua, Costa Rica, Panama, Colombia, St. Vincent, Paraguay, Argentina, Uruguay, Brazil: AM, PA, AC, MG, SP, RS, ES (Linhares, Aracruz, Colatina) (SILVA et al. 1968; MAES 1994; Malaguido \& PanizzI 1998; PanizzI et al. 2000b; CarPintero et al. 2014; Dellapé et al. 2015; Fernandes et al. 2015).

Material examined: (UFVB) $1 \hat{\sigma}$, Brasil - ES - Linhares, Graciosa II, Coord. 19¹7'43.17", 405'18.90", 11.III.2013, Col. M.C. Vélez. 1 సं, Brasil - ES - Aracruz, São José, Coord. 1941'9.49", 40¹7'19.36", 12.III.2013, Col. M.C. Vélez. 2 ㄱ, 2 o , Brasil - ES - Colatina, Sítio das Palmeiras, Coord. 19³5'57.95", 40³8'30.77", 14.III.2013, Col. M.C. Vélez.

Remarks: Specimens of $P$. guildinii were collected on pastures of Brachiaria brizantha, Panicum maximum cv. Mombaza.

\section{Pyrrhocoridae}

\section{Dysdercus maurus (Distant, 1901)}

Distribution: Colombia, Venezuela, Curaçao, Trinidad, Guyana, Suriname, Ecuador, Peru, Paraguay, Argentina, Brazil: AM, RN, PB, PE, BA, MG, RJ, SP, ES (Afonso Cláudio, São José do Calçado, Santa Teresa) (PeArson 1932; Doesburg 1968; Silva et al.1968; Froeschner 1981; Schaefer \& Ahmad 2000; Melo \& Dellapé 2013).

Material examined: (UFVB) $1 \hat{\jmath}$, Brasil - ES - Afonso Cláudio, Sítio Bom Sucesso, Coord. 20¹0'32.29", 41¹1'53.65", 05.III.2013, Col. M.C. Vélez. 3 స., Brasil - ES - São José do Calçado, Fazenda Vida Nova Soledade, Coord. 2058'19.81", 41³6'19.54", 28.II.2013, Col. M.C. Vélez. 1 ․, Brasil - ES - Santa Teresa, Fazenda Montanha, Coord. 1949'55.97", 4042'48.95", 08.III.2013, Col. M.C. Vélez.

Remarks: Specimens of $D$. maurus were collected on pastures of Brachiaria brizantha, Panicum maximum cv. Mombaza. Pastures were associated with weeds.

\section{Reduviidae}

\section{Apiomerus lanipes (Fabricius, 1803)}

Distribution: Mexico, Colombia, Argentina, Brazil: BA, SP, ES (Linhares, Pinheiros) (Amaral Filho et al. 1994; GiL-Santana \& Alencar 2001; Forero 2004; Marques et al. 2006; Coscarón \& MARTIN-PARK 2011; MARTINs et al. 2016).

Material examined: (UFVB) 1 specimen, Brasil - ES - Pinheiros, Fazenda São Roque, Coord. 18¹9'10.09", 40¹0'2.01", 19.III.2013, Col. M.C. Vélez.

Remarks: The specimen of $A$. lanipes was collected on pastures of Brachiaria brizantha.

\section{Atrachelus (Phorobura) saileri (Elkins, 1954)}

Distribution: Guyana, Suriname, Bolivia, Brazil AM, PA, ES* (Jerônimo Monteiro, Venda Nova do Imigrante, Itaguaçu, Santa Teresa) (ELKINS 1954).
Material examined: (UFVB) $1 \hat{\jmath}$, Brasil - ES - Jerônimo Monteiro, Fazenta Luzitania, Coord. 2046'10.64", 41 '23'4.32", 25.II.2013, Col. M.C. Vélez. 1 đ', Brasil - ES - Venda Nova, Sítio São José, Coord. 20²4'19.59", 41 7'1.47", 04.III.2013, Col. M.C. Vélez. (CEIOC) 1 specimen, Brasil - ES - Itaguaçu, Sítio Bela Vista, Coord. 19³7'14.03", 4051'45.12", 07.III.2013, Col. M.C. Vélez. 1 specimen, Brasil - ES - Santa Teresa, Sítio Natal, Coor. 1953'19.03", 4042'1.59", 08.III.2013, Col. M.C. Vélez.

Remarks: Specimens of $A$. (Phorobura) saileri were collected on pastures of Brachiaria brizantha, B. mutica, Cynodon spp. Panicum maximum cv. Mombaza.

\section{Cosmoclopius nigroannulatus (Stål 1860)}

Distribution: Venezuela, Bolivia, Argentina, Uruguay, Brazil: BA, RS, ES* (São José do Calçado, Baixo Guandu, Pinheiros) (Coscarón et al. 2002; JAHnKe et al. 2003; Melo et al. 2004; MARQues et al. 2006).

Material examined: (UFVB) $1 \hat{\lambda}$, Brasil - ES - São José do Calçado, Fazenda Soledade, Coord. 2057'19.19", 41 ³7'16.34", 28.II.2013, Col. M.C. Vélez. 1 đ̊, Brasil - ES - Pinheiros, Fazenda São Roque, Coord. 18¹9'10.09", 40¹0'2.01", 19.III.2013, Col. M.C. Vélez. (CEIOC) 2 specimens, Brasil - ES - Baixo Guandu, Sítio Seriema, Coord. 19³4'9.52", 4059'38.36", 14.III.2013, Col. M.C. Vélez.

Remarks: Specimens of $C$. nigroannulatus were collected on pastures of Brachiaria brizantha e B. decumbens.

\section{Doldina carinulata (Stål, 1859)}

Distribution: Venezuela, Guyana, Dominica, West Indies, Paraguay, Argentina, Brazil: AM, PA, MT, SP, ES* (Conceição do Castelo, Itaguaçu, Aracruz, João Neiva, Montanha, Barra de São Francisco) (Hussey \& Elkins 1955; Melo \& Coscarón 2004).

Material examined: (UFVB) 1 , Brasil - ES - Conc. Do Castelo, Sítio Montevideo, Coord. 20²8'17.04", 41¹5'37.22", 06.III.2013, Col. M.C. Vélez. 1 § , Brasil - ES - Itaguaçu, Corrego das flores, Coord. 1946'11.82", 4050'6.09", 07.III.2013, Col. M.C. Vélez. 1 त̂, Brasil - ES - Montanha, Fazenda Cinelândia, Coord. 187'16.91", 40¹5'32.49", 18.III.2013, Col. M.C. Vélez. 1 స., Brasil - ES - B. de São Francisco, Sítio dois corações, Coord. 18³7'17.99", 4053'8.53", 22.III.2013, Col. M.C. Vélez. (CEIOC) 1 specimen, Brasil - ES - Aracruz, São José, Coord. 1941'9.49", 40¹7'19.36", 12.III.2013, Col. M.C. Vélez. 1 specimen, Brasil ES - João Neiva, Três Ríos, Coord. 19³9'15.81", 40²5'25.36", 13.III.2013, Col. M.C. Vélez.

Remarks: Specimens of $D$. carinulata were collected on pastures of Brachiaria mutica, B. decumbens, Panicum maximum cv. Mombaza.

\section{Pnirontis infirma (Stål, 1859)}

Distribution: USA, Mexico, Cuba, Puerto Rico, Guatemala, Panama, Ecuador, Argentina, Brazil: RJ, ES* (Afonso Cláudio) (Champion 1897; Costa lima \& Seabra 1945; Wolkott 1948; Froeschner 1981; SWANSOn 2011; Diez \& Coscarón 2014).

Material examined: (CEIOC) 1 specimen, Brasil - ES - Afonso Cláudio, Fazenda Sermarv, Coord. 208'16.10", 418'19.19", 05.III.2013, Col. M.C. Vélez.

Remarks: The specimen of $P$. infirma was collected on pastures of Cynodon spp.

\section{Repipta flavicans (Amyot \& Serville, 1843)}

Distribution: Mexico, Cuba, Guatemala, Honduras, Nicaragua, Costa Rica, Panama, Colombia, Guyana, French Guiana, Bolivia, Paraguay, Argentina, Uruguay, Brazil: DF, 
RS, ES (São José do Calçado, Ecoporanga) (Melo et al. 2004; Barcellos 2006; Martin Park et al. 2012; Dellapé P. et al. 2015; GÁmeZ-VIRUÉs \& EBen 2005; MARTIns et al. 2016).

Material examined: (CEIOC) 1 specimen, Brasil - ES - São José do Calçado, Fazenda Vida Nova Soledade, Coord. 2058'19.81" 4136'19.54", 28.Il.2013, Col. M.C. Vélez. 1 specimen, Brasil - ES - Ecoporanga, Santa Teresinha, Coord. 18²9'20.89", 4049'45.38", 21.III.2013, Col. M.C. Vélez.

Remarks: Specimens of $R$. flavicans were collected on pastures of Cynodon spp. and Panicum maximum cv. Mombaza.

\section{Rhopalidae}

\section{Xenogenus picturatum (Berg, 1883)}

Distribution: USA, Mexico, Cuba, Puerto Rico, Nicaragua, St. Vincent, Bolivia, Argentina, Uruguay, Brazil: PR, ES* (Ecoporanga) (Maes \& Göllner-Scheiding 1993; Dellapé \& Carpintero 2012; Diez \& Coscarón 2015; Fowles et al. 2015; Melo \& MONTEMAYOR 2015).

Material examined: (MNRJ) 1 त, Brasil - ES - Ecoporanga, Sítio do Divino, Coord. 18¹9'33.39", 4048'52.69", 21.III.2013, Col. M.C. Vélez.

Remarks: The specimen of $X$. picturatum was collected on pastures of Brachiaria humidicola.

\section{Rhyparochromidae}

\section{Pseudopachybrachius vinctus (Say, 1831)}

Distribution: USA, Mexico, Cuba, Cayman Islands, Jamaica Bahamas, Dominican Republic, Puerto Rico, St. Croix Island, St. Thomas Island, Haiti, Belize, Guatemala, Panama, Antigua, Dominica, Barbados, Colombia, St. Vincent Island, Grenada, Guyana, Ecuador, Argentina, Brazil: MG, SP, RS, ES* (Atílio Vivacqua, Castelo, Venda Nova do Imigrante, Afonso Cláudio, Conceição do Castelo, Santa Teresa, Baixo Guandu, Vila Pavão) (Silva et al. 1968; Albuquerque 1990; Paula \& Ferreira 2000; Melo et al. 2004; BARANOWSKI \& SLATER 2005).

Material examined: (UFVB) 3 , Brasil - ES - Atílio Vivacqua,

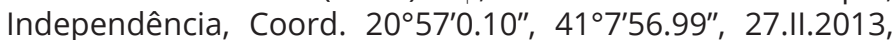
Col. M.C. Vélez. 1 + , Brasil - ES - Venda Nova, Sítio São José, Coord. 20²4'19.59", 417'1.47", 4.III.2013, Col. M.C. Vélez. 2 0, 1 q, Brasil - ES - Afonso Cláudio, Fazenda Sermarv, Coord. 208'16.10", 41ㅇ'19.19", 05.III.2013, Col. M.C. Vélez. 1 9, Brasil - ES - Conc. Do Castelo, Sítio Montevideo, Coord. 20²8'17.04", 41¹5'37.22", 6.III.2013, Col. M.C. Vélez. 3 q, Brasil - ES Santa Teresa, Sítio Natal, Coord. 1953'19.03", 4042'1.59", 08.III.2013, Col. M.C. Vélez. 1 , Brasil - ES - Vila Pavão, Sítio Alegre, Coord. 18³7'41.21", 4042'32.29", 20.III.2013, Col. M.C. Vélez. (USNM) 1 specimen, Brasil - ES - Castelo, Minerva, Coord. 20³1'2.50", 4110'47.25", 2.III.2013, Col. M.C. Vélez. 1 specimen, Brasil - ES - Baixo Guandu, Holz, Coord. 19³0'40.8", 410'1.54", 14.III.2013, Col. M.C. Vélez.

Remarks: Specimens of $P$. vinctus were collected on pastures of Brachiaria brizantha, B. decumbens, B. ruziziensis, and Cynodon spp.

\section{Thaumastocoridae}

\section{Thaumastocoris peregrinus (Carpintero \& Dellapé, 2006)}

Distribution: Australia, Kenya, Zimbabwe, South Africa, Chile, Argentina, Uruguay Brazil: MS, MG, RJ, SP, PR, SC, RS, ES (Castelo, Afonso Cláudio, Barra de São Francisco) (CARPINTERo \& Dellapé 2006; Martínez \& Bianchi 2010; Nadel et al. 2010; Wilcken et al. 2010; NOACK et al. 2011).

Material examined: (UFVB) 1 , Brasil - ES - Castelo,
Cachoeirinha de São José, Coord. 20³0'24.62", 41¹3'13.61", 02.III.2013, Col. M.C. Vélez. 1 đ̊, Brasil - ES - Afonso Cláudio, Sítio Bom Sucesso, Coord. 20¹0'32.29", 41¹1'53.65", 05.III.2013, Col. M.C. Vélez. 1 స̃, Brasil - ES - B. de São Francisco, Sítio Boa Vista, Coord. $18^{\circ} 35^{\prime 2} 28.79^{\prime \prime}, 40^{\circ} 53^{\prime} 30.28^{\prime \prime}, 22 . I I I .2013$, Col. M.C. Vélez.

Remarks: Specimens of Thaumastocoris peregrinus were collected on pastures of Brachiaria brizantha, Cynodon spp. Panicum maximum cv. Mombaza. In these places there were many remains (leaves) of eucalyptus trees, pastures were surrounded by this specie of tree.

\section{Tingidae}

\section{Gargaphia aff. Iunulata (Mayr 1865)}

Distribution: Colombia, Paraguay, Argentina, Uruguay, Brazil: PA, CE, BA, MG, RJ, ES* (Jerônimo Monteiro, Atílio Vivacqua) (Ajmat 2003; Coelho \& Silva 2015).

Material examined: (UFVB) 1 ก, 1 q, Brasil - ES - Jerônimo Monteiro, Sítio Boa Sorte, Coord. 2047'14.37", 41²4'43.08", 25.II.2013, Col. M.C. Vélez. 2 specimens, Brasil - ES Ecoporanga, Sítio Antenor Cavensan, Coord. 18²3'6.66", 4044'12.63", 21.III.2013, Col. M.C. Vélez. 1 `̃, Brasil - ES - Atílio Vivacqua, Independência, Coord. 2057'0.10", 41 '7'56.99", 27.II.2013, Col. M.C. Vélez.

Remarks: Specimens of $G$. lunulata were collected on pastures of Cynodon spp., Brachiaria brizantha, and Panicum maximum cv. Mombaza. Pastures were associated with weeds.

\section{DISCUSSION}

In the present study, forty-three species of Heteroptera belonging to thirteen families were collected on pastures of the Espírito Santo state: Alydidae (one specie), Berytidae (three species), Blissidae (one specie), Coreidae (seven species), Miridae (sixteen species), Nabidae (one specie), Pentatomidae (three species), Pyrrhocoridae (one species), Reduviidae (six species), Rhopalidae (one specie), Rhyparochromidae (one specie.), Thaumastocoridae (one specie), and Tingidae (one specie). Among the species collected; Cebrenis tenebrosa (Brailovsky, 1995) is recorded for the first time on Brazil, besides, the biogeographic range extension is also included for: N. parvus; G. nexus; J. macer; J. sobrinus; $B$. aff. antillus; $C$ duplicata; M. elevatus; V. sanctipauli; V. westwoodi; C. infumatus; C. femoralis; D. linearis; E. varians; G. gracilentus; $M$. praeclarus; $P$. quadrimaculatus; S. carmelitana; T. apicalis; T. tenuis; $T$. cucurbitaceus; N. capsiformis; D. melacanthus; O. ypsilongriseus; A. (Phorobura) saileri; P. infirma; C. nigroannulatus; D. carinulata; $P$. infirma; $X$. picturatum; $P$. vinctus; $G$. aff. lunulata. This study showed the highest diversity of heterpterans on patures ecosystems of Espírito Santo state.

The vegetational diversity and density in agroecosystems surrounding the pastures influenced the abundance of herbivorous insects as well as their natural enemies (AnDow 1983; SCHELLHORN \& SORK 1997). We believe that the association of pastures with weeds and the presence of crop residues improved the records of this study.

Among the forty-three collected species of Heteroptera three of them were observed inducing extensive damage and killing most of the pastures leaves. The species $B$. aff. antillus; C. oleosa and $T$. tenuis were collected in many pastures, although only in Tangola grass (Brachiaria mutica $\times$ Brachiaria arrecta); Angola grass (B. mutica) and Star grass (Cynodon sp.), respectively were observed causing serious damage. Besides, O. ypsilongriseus was observed feeding of developing grains of bread grass (B. brizantha). 
The plant bugs: B. aff. antillus; C. oleosa, T. tenuis, and $O$. ypsilongriseus are important sapsuckers, those are specialized for feeding especially on Poaceae family plants (PanizzI et al. 2000b; ValÉRIO et al. 2015; Morales et al. 2016; Vivas \& Astudillo 2017). The damages produced by the first three species before mentioned are similar, the youngest leaves attacked can partially or dry out, delaying the growth, reducing yield and nutritional value of the crops and grasses palatability; elsewhere, when the damage of insect is serious its may cause plants death (Valério et al. 2015; Vivas \& Astudillo 2017; Vélez et al. 2020). In the case of 0 . ypsilongriseus, is considered an important pest of rice in South America, adults and nymphs feeding kernels cause conspicuous areas of discoloration, panicles can be partially or empty and therefore causing losses in the yield and quality of the grain (PANIzzI et al. 2000b; VIVAS \& Astudillo 2011). The damages of O. ypsilongriseus on pastures seeds need to receive more attention, especially to small cattle breeders that depend on good seeds condition to keep their grasslands.

Therefore, this study helps to increase knowledge about the relationships between heteropterans and pastures. Besides, the timely and accurate identification of heteropterans on pastures could help to recognize among beneficial and harmful insects; establish management strategies to pest insects; prevent the pasture degradation attributed to pest damages, and consequently to avoid losses of dry matter, grass digestibility, which are directly related on the reduction of milk as well as the meat production. Finally, more efforts are still necessary to increase the number of heteropterans species known on the state.

\section{ACKNOWLEDGMENTS}

The authors would like to thank the Conselho Nacional de Desenvolvimento Científico e Tecnológico (CNPq) and the Coordenação de Aperfeiçoamento de Pessoal de Nível Superior (Capes) for the financial support. We are very grateful to Carl W. Schaefer (in memory), University of Connecticut, Entomological Collection, Storrs, USA), Thomas J. Henry (National Museum of Natural History, Washington, D.C., USA), Hélcio Reinaldo Gil Santana (Instituto Oswaldo Cruz, RJ, Brazil), Aline Barcellos (Museu de Ciências Naturais da Fundação Zoobotânica do Rio Grande do Sul, RS, Brazil), Luiz Antônio Alves Costa (Museu Nacional, Universidade Federal do Rio de Janeiro, RJ, Brazil), Harry Brailovsky (Universidad Nacional Autónoma de México, Departamento de Zoologia, D.F., México) to identify several specimens collected, to Sistema Integrado de Bases Geoespaciais do Estado do Espírito Santo (GEOBASES) particularly to Samuel Martins da Costa Coura for proceessing the data used to construct the map showing the sampling sites, to every single person from the Instituto Capixaba de Pesquisa Assistência Técnica e Extensão Rural (INCAPER) at Espírito Santo for all their support, and Espírito Santo cattle breeders that allowed us to make the collection of heteropterans on their properties. Finally, we would like to thank Irina Morales (Universidad Pedagógica y Teconológica de Colombia) for reading previous versions of this paper and for suggesting improvements.

\section{REFERENCES}

Ajmat, MV, SG Bado, MA Coviella \& MJ Pannunzio, 2003. Aspectos morfológicos, biológicos y daño de Gargaphia lunulata (Mayr) 1865 (Heteroptera: Tingidae) sobre Passiflora caerulea L. (Passifloraceae). Boletin de Sanidad Vegetal - Plagas, 29: 339-346.

Albuquerque, GS,1990. Ocorrência de Cymoninus notabilis (Distant) e Pseudopachybrachius vincta (Say) (Hemiptera: Lygaeidae) na cultura do arroz no Rio Grande do Sul. Anais da Sociedade Entomológica do Brasil, 20: 217-218.

Almeida, JEM, AB Filho, VA Costa, LG Leite, ZA Ramiro, PC
Kruppa \& EMB Calil, 2000. Manejo Integrado de pragas e doenças das pastagens. Sao Paulo, Secretaria de Agricultura e abastecimento.

Amaral Filho, BF, I Gióia, CM Waib, E Mendeleck \& FL Cônsoli, 1994. Observações sobre biologia de Apiomerus lanipes (Fabricius) (Hemiptera, Reduviidae). Revista Brasileira de Zoologia, 11: 283-288. DOI: https://doi.org/10.1590/s010181751994000200012

Andow, DA, 1983. Effect of agricultural diversity on insect populations, pp 91-115. In: Lockeretz, W (Ed.). Environmentally sound agriculture. Praeger, New York.

Baranowski, RM \& JA Slater, 2005. The Lygaeidae of the West Indies. Florida Agricultural Experiment Stations Bulletin, 402: 1-266.

Barcellos, A, 2006. Hemípteros terrestres, pp. 198-209. In: Becker, FG, RA Ramos \& LA Moura, (Eds.). Biodiversidade. Regiões da Lagoa do Casamento e dos Butiazais de Tapes, planície costeira do Rio Grande do Sul. Brasília, Ministério do Meio Ambiente.

Barcellos, A, LS Schmidt \& PSF Ferreira, 2011. Composition and structure of a Miridae (Hemiptera, Heteroptera) assemblage from a relict of deciduous rainforest in southern Brazil. Iheringia. Série Zoologia, 101: 115-120. DOI: https://doi.org/10.1590/s0073-47212011000100016

Blöte, HC, 1938. Catalogue of the Coreidae in the Rijksmuseum van Natuurlijke Histoire Part IV. Coreinae, Third Part, Zoologische Mededeelingen, 20: 275-308

Brailovsky, H, 1995. Revisión del género Cebrenis (HemipteraHeteroptera-Coreidae-Coreini). Anales del Instituto de Biología. Universidad Nacional Autónoma de México. Publicaciones especiales 15.

Brailovsky, H, 2009. Revision of the Phthia complex with a description of four new genera (Hemiptera: Heteroptera: Coreidae: Coreinae: Leptoscelini). Acta Entomologica Musei Nationalis Pragae, 49: 59-74.

Carpintero, DL \& PM Dellapé, 2006. A new species of Thaumastocoris Kirkaldy from Argentina (Heteroptera: Thaumastocoridae: Thaumastocorinae). Zootaxa, 1228: 61-68. DOI: https://doi.org/10.11646/zootaxa.1228.1.4

Carpintero, DL, S De Biase \& SA Konopko, 2014. HemipteraHeteroptera de la Reserva Ecológica Costanera Sur (Ciudad Autónoma de Buenos Aires), Argentina. Revista del Museo Argentino de Ciencias Naturales, 16: 67-80. DOI: https://doi.org/10.22179/revmacn.16.162

Carvalho, JCM \& AV Fontes, 1981. Mirídeos neotropicais CCXXV: Revisão do gênero Collaria Provancher no continente Americano (Hemiptera). Experientiae, 27: 11-46.

Carvalho, JCM \& CRS Afonso, 1977. Mirideos neotropicais, CCVIII: Sobre uma coleção enviada para estudo pela Academia de Ciências da Califórnia (Hemiptera). Revista Brasileira de Biologia, 37: 7-16. DOI: https://doi.org/10.1590/s0101-81751987000100002

Carvalho, JCM \& J Jurberg, 1976. Neotropical Miridae, CCVI: Revisão do gênero Horciasinus Carvalho \& Jurberg (Hemiptera). Revista Brasileira de Biologia, 36: 811-834.

Carvalho, JCM \& LAA Costa, 1988. Mirideos neotropicais, CCXCII: Revisão do gênero Sericophanes Reuter na America do Sul e America Central (Hemiptera). Revista Brasileira de Biologia, 48: 897-909.

Carvalho, JCM, 1944. Mirídeos Neotropicais: Sobre o gênero "Sericophanes" Reuter, com descrição de uma nova espécie (Hemiptera). Revista Brasileira de Biologia, 4: 517-530.

Carvalho, JCM, 1945. Mirídeos neotropicais, XIX: Gênero Macrolophus Fieber, com descrição de duas espécies novas e Solanocoris n. g. (Hemiptera). Revista Brasileira de Biologia, 5: 525-534.

Carvalho, JCM, 1947. Mirideos Neotropicais, XXVII: Generos Porpomiris Berg, Lampethusa Distant, Cyrtopeltis Fieber e Dycyphus Fieber (Hemiptera). Boletim do Museu Nacional, 77: 1-23.

Carvalho, JCM, 1948. Mirideos neotropicais, 30: Gêneros 
Ellenia Reuter, Eurychilopterella Reuter, e Rhinacloa Reuter, com descrições de espécies novas (Hemiptera). Boletim do Museu Nacional (n. s.) (Zoologia), 85: 13.

Carvalho, JCM, 1975a. Neotropical Miridae, CLXXXVIII: On the genera Dolichomiris Reuter, Megaloceroea Fieber, Stenodema Laporte, Trigonotyliscus n. gen. and Trigonotylus Fieber (Hemiptera). Revista Brasileira de Biologia, 35: 121 140.

Carvalho, JCM, 1975b. Mirideos neotropicais, CXCIII: Sôbre algumas espécies que ocorrem nas caatingas brasileiras (Hemiptera). Revista Brasileira de Biologia, 35: 451-459.

Champion, GC, 1897. Insecta: Rhynchota (HemipteraHeteroptera). Vol. II. pp. 162-296. In: Goodwin, F.O. and Salvin, O. (Eds.). Biologia Centrali-Americana. London.

Chocorosqui, VR \& AR Panizzi, 2004. Impact of cultivation systems on Dichelops melacanthus (Dallas) (Heteroptera: Pentatomidae) population and damage and its chemical Control on wheat. Neotropical Entomology, 33: 487-492. DOI: https://doi.org/10.1590/S1519-566X2004000400014

Coelho, LBN \& ER Da-Silva, 2015. Registro de dano de Gargaphia lunulata (Insecta: Hemiptera: Tingidae) em Passiflora edulis (Malpighiales: Passifloraceae) e Arachis repens (Fabales: Fabaceae) em uma área urbana do Rio de Janeiro. Agrarian Academy, 2: 90-100. DOI: https://doi.org/10.18677/agrarian_academy_009

Cornelis, M \& MC Coscarón, 2013. The Nabidae (Insecta, Hemiptera, Heteroptera) of Argentina. Zookeys, 333: 1-30. DOI: https://doi.org10.3897/zookeys.333.5084

Coscarón, MC \& A Martin-Park, 2011. New data for Argentinian Assassin Bugs Fauna (Hemiptera: Reduviidae: Harpactorinae). Munis Entomology \& Zoology, 6: 758-763.

Coscarón, MC, MC Melo \& DV Ohashi, 2002. Description of the egg and fourth instar of Cosmoclopius nigroannulatus (Stål) (Heteroptera, Reduviidae, Harpactorinae). Facena, 18: 59-61.

Costa Lima, A, 1940. Insetos do Brasil. Vol. 2. Hemípteros. Escola Nacional de Agronomia. Série didática no. 3, Rio de Janeiro, Brazil.

Costa Lima, AMC \& CAC Seabra, 1945. Stenopodinae da coleção do Instituto Oswaldo Cruz (Hemiptera: Reduvioidea: Reduviidae) (2a nota). Memórias do Instituto Oswaldo Cruz, 42(2): 287-292.

Cruces, L \& C Vergara, 2015. Distribución de las especies de ocho géneros de la tribu Coreini (Heteroptera: Coreidae) en Perú. Ecología Aplicada, 14: 1-20. DOI: https://doi.org/10.21704/rea.v14i1-2.80

Dellapé, G, DA Rider \& PM Dellapé, 2015. Notes on distributions for Argentinean Pentatomidae (Heteroptera: Pentatomoidea), with new records in the country. Revista Brasileira de Entomologia, 59: 169-176. DOI: https://doi.org/10.1016/j.rbe.2015.06.001

Dellapé, PM \& DL Carpintero, 2012. Relevamiento de los Heteroptera (Insecta: Hemiptera) de las Sierras de Tandil, provincia de Buenos Aires, Argentina. Revista del Museo Argentino de Ciencias Naturales, 14: 125-134.

Dellapé, PM \& TH Henry, 2020. SF Lygaeoidea: Lygaeoidea Species File (version 5.0, Jun 2018). In: Species 2000 \& ITIS Catalogue of Life, 2020-02-24 (Roskov Y, G Ower, T Orrell T, D Nicolson, N Bailly, PM Kirk, T Bourgoin, RE DeWalt, W Decock, E Nieukerken, L Penev. Available on: $<$ http://www.catalogueoflife.org/col>. Species 2000: Naturalis, Leiden, the Netherlands.

Dellapé, PM, DL Carpintero \& MC Melo, 2010. New records of Dipsocoromorpha, Cimicomorpha and Pentatomomorpha (Hemiptera: Heteroptera) from Argentina. Zootaxa, 2436: 57-64. DOI: https://doi.org/10.11646/zootaxa.2436.1.3

Dellapé, PM, MC Melo, SI Montemayor, G. Dellapé \& H Brailovsky, 2015. Terrestrial Heteroptera (Hemiptera) from Moconá Provincial Park (Misiones, Argentina). Check List, 11(3): 1662. DOI: https://doi.org/10.15560/11.3.1662

Dias-Filho, MB, 2011. Degradação de pastagens: processos, causas e estratégias de recuperação. 4. ed. Belém, PA. Embrapa Amazônia Oriental.

Diez, F \& MC Coscarón, 2014. The Stenopodainae (Hemiptera, Heteroptera) of Argentina. ZooKeys 452: 51-77. DOI: https://doi.org/10.3897/zookeys.452.6519

Diez, F \& MC Coscarón, 2015. Contribution to the knowledge of Patagonia, Argentina: redescription of the genus Xenogenus Berg 1883 (Hemiptera: Heteroptera: Rhopalidae) and description of immature stages of Xenogenus gracilis Reed, 1899. Zootaxa, 3919: 573-582. DOI: https://doi.org/10.11646/zootaxa.3919.3.7

Doesburg, Jr.PH Van, 1968. A revision of the New World species of Dysdercus Guérin Méneville (Heteroptera: Pyrrhocoridae). Zoologische Verhandelingen, 97: 1-215.

Dolling, WR, 1977. A revision of the Neotropical genus Vilga Stål (Hemiptera: Coreidae). Systematic Entomology, 2: 2744.

Elkins, JC, 1954. A synopsis of Atrachelus (Hemiptera, Reduviidae). Proceedings of the Entomological Society of Washington, 56: 97-120.

ESRI, 2010. ArcGIS ArcMap, desktop GIS software. Redlands, CA: ESRI Inc. Version 10.0. Available on: <https:// support.esri.com/es/products/desktop/arcgis-desktop/ arcmap/10>

Euclides, VPB, CB Valle, MCM Macedo, RG, Almeida, DB Montagner \& RA Barbosa, 2010. Brazilian scientific progress in pasture research during the first decade of XXI century. Revista Brasileira de Zootecnia, 39: 151-168. DOI: https://doi.org/10.1590/s1516-35982010001300018

Fernandes, JAM, PL Mitchell, L Livermore \& M Nikunlassi, 2015. Leaf-Footed Bugs (Coreidae), pp 548-605. In: Panizzi A.R. and Grazia J. (Eds.). The true bugs (Heteroptera) of the Neotropics, Springer.

Ferreira, PSF \& TJ Henry, 2011. Synopsis and keys to the tribes, genera, and species of Miridae (Hemiptera: Heteroptera) of Minas Gerais, Brazil. Part I: Bryocorinae. Zootaxa, 2920: 1-41. DOI: https://doi.org/10.11646/zootaxa.2920.1.1

Ferreira, PSF, 1980. Taxonomia das espécies do genero Polymerus Hahn, 1831 (Hemiptera, Miridae) da região Cisandina, América do Sul. Experientae, 26: 329-385.

Ferreira, PSF, ER Silva \& LBN Coelho, 2001. Miridae (Heteroptera) fitófagos e predadores de Minas Gerais, Brasil com ênfase em espécies com potencial econômico. Iheringia. Série Zoologia, 91: 159-169. DOI: https://doi.org/10.1590/s0073-47212001000200022

Ferreira, PSF, TJ Henry \& LA Coelho, 2015. Plant Bugs (Miridae), pp. 237-286. In: Panizzi, AR \& J Grazia (Eds.). True Bugs (Heteroptera) of the Neotropics, Entomology in Focus.

Forero, D, 2004. Diagnosis de los géneros Neotropicales de la familia Reduviidae (Hemiptera: Heteroptera) y su distribución en Colombia (excepto Harpactorinae), pp 129275. In: Fernández, F, G Andrade \& G Amat (Eds.). Insectos de Colombia, Vol. 3. Universidad Nacional de Colombia: Bogotá.

Forero, D, 2006. New Heteroptera (Insecta: Hemiptera) records from Colombia. Caldasia, 28(1): 125-128.

Fowles, TM, MC Coscarón, AR Panizzi \& SP Carroll, 2015. Scentless Plant Bugs (Rhopalidae), pp 607-637. In: Panizzi, AR \& J Grazia (Eds.). The true bugs (Heteroptera) of the Neotropics, Springer.

Froeschner, RC, 1981. Heteroptera or true bugs of Ecuador: A partial catalog. Smithsonian Contributions to Zoology 322: 147. DOI: https://doi.org/10.5479/si.00810282.322

Gámez-Virués, S \& A Eben, 2005. Predatory behavior of Repipta flavicans Stål (Hemiptera: Reduviidae), a natural enemy of Diabroticina (Coleoptera: Chrysomelidae). Proceedings of the Entomological Society of Washington, 107: 642-651.

Gil-Santana, HR \& J Alencar, 2001. Reduviidae da Reserva Florestal de Linhares, Espírito Santo, Brasil (HemipteraHeteroptera). Revista Brasileira de Zoociências, 3: 185194. 
Grazia, J, \& JAM Fernandes, 2012. Subordem Heteroptera Linnaeus, 1758, pp. 369-405. In: Rafael, JA, GAR Melo, CJB Carvalho, SA Casari \& R Constantino (Eds.). Insetos do Brasil. Diversidade e Taxonomia. Ribeirão Preto: Holos Editora.

Grazia, J, 1978. Revisão do gênero Dichelops Spinola, 1837 (Heteroptera, Pentatomidae, Pentatomini). Iheringia. Série Zoologia, 53: 3-119.

Gullan, PJ \& PS Cranston, 2014. The Insects: An Outline of Entomology. John Wiley \& Sons, Ltd.

Henry, TJ \& MR Wilson, 2004. First records of eleven true bugs (Hemiptera: Heteroptera) from the Galápagos Islands, with miscellaneous notes and corrections to published reports. Journal of the New York Entomological Society, 112: 75-86. DOI: https://doi.org/10.1664/0028-7199(2004)112[0075:fr oetb]2.0.co;2

Henry, TJ \& RC Froeschner, 1998. Catalog of the Stilt bugs, or Berytidae, of the World (Insecta: Hemiptera: Heteroptera). Contributions of the American Entomological Institute, 30: $1-72$.

Henry, TJ, 1997. Monograph of the stilt bugs, or Berytidae (Heteroptera), of the Western Hemisphere. Memoirs of the Entomological Society of Washington, 19: 1-149.

Henry, TJ, 2009. Biodiversity of Heteroptera, pp 223-263. In: Foottit, R \& P Adler (Eds.). Insect Biodiversity: Science and Society. Blackwell Publishing.

Henry, TJ, 2012. Revision of the plant bug genus Tytthus (Hemiptera, Heteroptera, Miridae, Phylinae). ZooKeys, 220: 1-114. DOI: https://doi.org/10.3897/zookeys.220.2178

Hernández, LM \& TJ Henry, 2010. The Plant Bugs, or Miridae (Hemiptera: Heteroptera), of Cuba. Pensoft Publishers, Sofia and Moscow.

Hussey, RF \& JC Elkins, 1955. Review of the genus Doldina Stål (Hemiptera, Reduviidae). Quarterly Journal of the Florida Academy of Science, 18: 261-278.

IBGE - Instituto Brasileiro de Geografia e Estatística, 2006. Censo Agropecuário 2006. Série AGRO03. Utilização das terras (ha). Available on: http://biblioteca.ibge.gov.br/ visualizacao/periodicos/51/agro_2006.pdf. [Acessed in: 11.x. 2018.

Jahnke, SM, LR Redaelli, LMG Diefenbach \& CFS Efrom, 2003. Distribuição espacial de posturas de Cosmoclopius nigroannulatus Stål (Hemiptera: Reduviidae) em Nicotiana tabacum L. (Solanaceae). Neotropical Entomology, 32: 123-126. DOI: https://doi.org/10.1590/s1519$566 \times 2003000100018$

Kellogg, EA, 2001. Evolutionary history of the grasses. Plant Physiology, 125: 1198-1205. DOI: https://doi.org/10.1104/ pp.125.3.1198

Kormilev, NA, 1954. Notas sobre Coreidae neotropicales II; (Hemiptera Merocorinae de la Argentina y paíises limítrofes. Revista Ecuatoriana de Entomologia y Parasitologia. 2: 153-187.

Lani, JL, M Resende, SB Resende \& LR Feitoza, 2008. Atlas dos Ecossistemas do Espírito Santo. SEMA, UFV.

Maes, JM \& U Göllner-Scheiding, 1993. Catálogo de los Coreoidea (Heteroptera) de Nicaragua. Revista Nicaragüense de Entomología, 25: 1-19.

Maes, JM, 1994. Catálogo de los Pentatomoidea (Heteroptera) de Nicaragua. Revista Nicaraguense de Entomología, 28: 1-29.

Malaguido, AB \& AR Panizzi, 1998. Pentatomofauna associated with sunflower innorthern Parana State, Brazil. Anais da Sociedade Entomológica do Brasil, 27: 473-475. DOI: https://doi.org/10.1590/s0301-80591998000300017

Marques, OM, HR Gil-Santana, ML Coutinho \& DD Silva Júnior, 2006. Percevejos predadores (Hemiptera, Reduviidae, Harpactorinae) em fumo (Nicotiana tabacum L.) no município de Cruz das Almas, Bahia. Revista Brasileira de Zoociências, 8: 55-60.59.

Martínez, AM, M Baena, Jl Figueroa, P Del Estal, M Medina,
E Guzmán-Lara \& S Pineda, 2014. Primer registro de Engytatus varians (Distant) (hemiptera: Heteroptera: Miridae) en México y su depredación sobre Bactericera cockerelli (Šulc) (Hemiptera: Triozidae): una revisión de su distribución y hábitos. Acta Zoológica Mexicana, 30: 617624. DOI: https://doi.org/10.21829/azm.2014.30381

Martínez, G \& M Bianchi, 2010. Primer registro para Uruguay de la chinche del eucalipto, Thaumastocoris peregrinus Carpintero y Dellapé, 2006 (Heteroptera: Thaumastocoridae). Agrociencia, 14: 15 -18.

Martins DS, PSF Ferreira, MJ Fornazier \& JS Santos, 2016. Coleópteros e hemípteros da Reserva Natural Vale, pp. 341-363. In: Rolim SG, LFT Menezes \& AC Srbek-Araujo (Eds). Floresta Atlântica de Tabuleiro: diversidade e endemismos na Reserva Natural Vale. Belo Horizonte, Editora Rupestre.

Melo, MC \& PM Dellapé, 2013. Catalogue of the Pyrrhocoroidea (Hemiptera: Heteroptera) from Argentina. Revista de la Sociedad Entomológica Argentina, 72: 55-74.

Melo, MC \& SI Montemayor, 2015. Biodiversity of the scentless plant bugs (Hemiptera: Rhopalidae) in southern South America. Journal of Natural History, 1-38. DOI: https://doi.org/10.1080/00222933.2015.1073810.

Melo, MC \&. MC Coscarón, 2004. New records of Reduviidae (Hemiptera: Heteroptera) from Argentina. Zootaxa, 698: 1-4. DOI: https://doi.org/10.11646/zootaxa.698.1.1

Melo, MC, PM Dellapé, DL Carpintero \& MC Coscarón, 2004. Reduviidae, Miridae y Lygaeoidea (Hemiptera) recolectados en Colonia Carlo Pellegrini (Esteros de Iberá, Corrientes, Argentina). Revista de la Sociedad Entomológica Argentina, 63: 59-67.

Menezes, M, 1990. Collaria oleosa (Distant, 1883) (Hemiptera: Miridae), nova praga de gramíneas forrageiras no sudeste de Bahia, Brasil. Agrotrópica Olhéus, 2: 113-118.

Morales, I, PSF Ferreira \& D Forero, 2016. Taxonomic revision of Collaria Provancher, 1872 (Hemiptera: Miridae) with the description of a new species from the Afrotropical region. Zootaxa, 4138: 201-246. DOI: https://doi.org/10.11646/ zootaxa.4138.2.1

Nadel, RL, B Slippers, MC Scholers, SA Lawson, AE Noack, CF Wilcken, JP Bouvet \& MJ Wingfield, 2010. DNA bar-coding reveals source and patterns of Thaumastocoris peregrinus invasions in South Africa and South America. Biological Invasions, 12:1067-1077. DOI: https://doi.org/10.1007/ s10530-009-9524-2

Nardi, C, PM Fernandes, OD Rodriguez \& JMS Bento, 2007. Flutuação populacional e distribuição vertical de Scaptocoris carvalhoi Becker (Hemiptera: Cydnidae) em Área de Pastagem. Neotropical Entomology, 36: 107-111. DOI: https://doi.org/10.1590/s1519-566x2007000100013

Noack, AE, G Cassis \& HA Rose, 2011. Systematic revision of Thaumastocoris Kirkaldy (Hemiptera: Heteroptera: Thaumastocoridae). Zootaxa, 3121: 1-60. DOI: https://doi.org/10.11646/zootaxa.3121.1.1

Ojeda-Peña, D, 1971. Biología y hábitos de Nabis capsiformis Germar (Hemip.: Nabidae). Sociedad Entomológica de Perú, 14: 297-303.

Oliveira, LJ \& AB Malaguido, 2004. Flutuação e distribuição vertical da população do percevejo castanho-da-raiz, Scaptocoris castanea Perty (Hemiptera: Cydnidae), no perfil do solo em áreas produtoras de soja nas regiões centro-oeste e sudeste do Brasil. Neotropical Entomology, 33: 283-291. DOI: https://doi.org/10.1590/s1519$566 \times 2004000300002$

Olivera, L, MC Melo \& PM Dellapé, 2017. First records of leaffooted bugs (Heteroptera: Coreoidea: Coreidae) from Argentina. Revista de la Sociedad Entomológica Argentina, 76: 68-70. DOI: https://doi.org/10.25085/rsea.761212

Panizzi, AR \& J Grazia, 2015. Introduction to True Bugs (Heteroptera) of the Neotropics, pp. 3-20. In: Panizzi, AR \& J Grazia (Eds). True Bugs (Heteroptera) of the Neotropics, 


\section{Springer.}

Panizzi, AR, AF Bueno, FAC Da Silva, 2013. Insetos que atacam vagens e graos, pp. 493-629. In: Hoffman-Campo CB, BS Correia-Ferreira \& F Moscardi (Eds.) Soja: manejo integrado de insetos e outros artrópodes-praga. Embrapa, Brasília.

Panizzi, AR, CW Schaefer \& Y Natuhara, 2000a. Broad-headed bugs (Alydidae), pp. 321-336. In: Schaefer CW \& AR Panizzi (Eds.). Heteroptera of economic importance. CRC Press, Boca Raton.

Panizzi, AR, CW Schaefer, 2015. Broad-headed bugs (Alydidae), pp. 537-547. In: Panizzi AR \& J Grazia (Eds.). The true bugs (Heteroptera) of the Neotropics, Springer.

Panizzi, AR, JE McPherson, DG James, M Javahery \& RM McPherson, 2000b. Stink bugs (Pentatomidae), pp. 421474. In: Schaefer CW \& AR Panizzi (Eds). Heteroptera of economic importance. CRC Press, Boca Raton.

Paula, AS \& PSF Ferreira, 2000. Fauna de Heteroptera de La "Mata Do Córrego do Paraíso", Viçosa, Minas Gerais, Brasil. II. Patrones Temporales. Distribución Anual y Estacionalidad. Anales del Instituto de Biología de la Universidad Nacional Autónoma de México. Serie Zoología, 7: 7-19.

Pearson, EO, 1932. Notes on the genus Dysdercus (Hemiptera- Heteroptera) in Trinidad, B. W. I. Psyche : A Journal of Entomology, 39: 113-126. DOI: https://doi.org/10.1155/1932/71305

Rebagliati, P, LM Mola, AG Papeschi \& J Grazia, 2005. Cytogenetic studies in Pentatomidae (Heteroptera): A review. Journal of Zoological Systematics and Evolutionary Research, 43: 199-213. DOI: https://doi.org/10.1111/ j.1439-0469.2005.00312.x

Rengifo-Correa, LA\& R González, 2011. Lygaeoidea (Hemiptera: Heteroptera) de Parques Nacionales Naturales (pnn) con nuevos registros para Colombia. Revista Colombiana de Entomología, 37: 331-340.

Sailer, RI, 1944. The genus Solubea (Heteroptera: Pentatomidae). Proceedings of the Entomological Society of Washington, 46: 105-127.

Samuels, RI \& DAL Coracini, 2004. Selection of Beauveria bassiana and Metarhizium anisopliae isolates for the control of Blissus antillus (Hemiptera: Lygaeidae). Scientia Agricola, 61: 271-5. DOI: https://doi.org/10.1590/s010390162004000300005

Samuels, RI, DLA Coracini, CA Martins dos Santos \& CAT Gava, 2002. Infection of Blissus antillus (Hemiptera: Lygaeidae) Eggs by the entomopathogenic fungi Metarhizium anisopliae and Beauveria bassiana. Biological Control, 23: 269-273. DOI: https://doi.org/10.1006/bcon.2001.1009

Schaefer, CW \& AR Panizzi, 2000. Economic Importance of Heteroptera: A General View, pp. 3-8. In: Schaefer CW \& Panizzi AR (Eds.). Heteroptera of economic importance. CRC Press, Boca Raton.

Schaefer, CW \& I Ahmad, 2000. Cotton strainers and their relatives (Pyrrhocoroidea: Pyrrhocoridae and Largidae), pp. 271-308. In: Schaefer CW \& AR Panizzi (Eds.). Heteroptera of economic importance. CRC Press, Boca Raton.

Schaefer, CW \& I Ahmad, 2008. A revision of Neomegalotomus (Hemiptera: Alydidae). Neotropical Entomology, 37: 30-44.

Schellhorn, NA \& VL Sork, 1997. The impact of weed diversityon insect population dynamics and crop yield in collards, Brassica oleracea (Brassicaceae). Oecologia, 111: 233-240. DOI: https://doi.org/10.1007/s004420050230

Schuh, RT \&JA Slater, 1995. True Bugs of the World (Hemiptera: Heteroptera), Classification and Natural History. Cornell \& Comstock Publishing Associates.

Schuh, RT \& MD Schwartz, 1988. Revision of the New World Pilophorini (Heteroptera: Miridae: Phylinae). Bulletin of the American Museum of Natural History, 187: 101-201.

Schuh, RT, 2002-2013, On-line Systematic Catalog of Plant Bugs (Insecta: Heteroptera: Miridae). Available on: <http://research.amnh.org/pbi/catalog/>. [Accessed in: 14.vi.2020].

Silva, AGA, CR Gonçalves, DM Galvão, AJL Gonçalves, J Gomes, MN Silva \& L De Simoni, 1968. Quarto catálogo dos insetos que vivem nas plantas do Brasil. Seus parasitos e predadores. Parte II, 1. Ministerio da Agricultura, Rio de Janeiro.

Silva, DB, RT Alves, PSF Ferreira \& AJA Camargo, 1994. Collaria oleosa (Distant, 1883) (Heteroptera: Miridae), Uma Praga Potencial na Cultura do Trigo na Região dos Cerrados. Pesquisa Agropecuária Brasileira, 29: 2007-2012.

Stål, C, 1870. Enumeratio Hemipterorum. Bidrag Hill en företeckning öfver alla hittills kända Hemiptera, jemte systematiska meddelanden. Pt. 1. Svenska Vetenskaps-Akademiens Handlingar, 9: 1-232. DOI: https://doi.org/10.5962/bhl.title.12549

Swanson, DR, 2011. New state records and distributional notes for some assassin bugs of the continental United States. The Great Lakes Entomologist, 44: 117-138.

Thum, AB \& EC Costa, 1997. Coreidae (Heteroptera) associados a espécies florestais. Ciencia. Florestal, 7: 27-31.

Valério, JR, JM Vieira \& LCS Valle, 1999. Ocorrência de Blissus antillus Leonard (Hemiptera: Lygaeidae: Blissinae) em pastagem no Estado de Mato Grosso do Sul. Anais da Sociedade Entomológica do Brasil, 28: 527-529. DOI: https://doi.org/10.1590/s0301-80591999000300020

Valério, JR, PR Reis \& JO Lima, 2015. Percevejo-das-gramíneas, Blissus ?leucopterus, pp. 708-719. In: Vilela, EF \& RA Zucchi (Eds.). Pragas introduzidas no Brasil: insetos e ácaros. Piracicaba: FEALQ.

Vélez, M, G Dias, I Morales, RNC Guedes \& J Lino-Neto, 2020. Morphology and histology of the male reproductive system of Collaria oleosa (Distant, 1883) (Heteroptera: Miridae). Bionatura, 5: 1122-1125. DOI: https://doi.org/10.21931/ $\mathrm{rb} / 20120.05 .02 .7$

Vivan, LM, C Nardi, J Grazia \& JM Bento, 2013. Description of the immatures of Scaptocoris carvalhoi Becker (Hemiptera: Cydnidae). Neotropical Entomology, 42: 288292. DOI: https://doi.org/10.21931/rb/20120.05.02.7

Vivas, CLE \& D Astudillo, 2011. Plantas hospederas de chinche vaneadora en el cultivo de arroz en Calabozo, Estado Guárico, Venezuela. Agronomía Tropical, 60: 369-373.

Vivas, CLE \& DH Astudillo, 2017. Fluctuación poblacional de Trigonotylus tenuis en arroz en Calabozo Estado Guárico, Venezuela. Journal of the Selva Andina Biosphere, 5: 8093. DOI: https://doi.org/10.36610/j.jsab.2017.050200080

Vivas, LC, M Cermeli \& F Godoy, 2005. Primera cita de Trigonotylus tenuis Reuter, 1893 (Hemiptera: Miridae) causando daños en el cultivo del arroz (Oryza sativa L.) en Venezuela. Entomotropica, 20: 125-126.

Weirauch, C \& RT Schuh, 2011. Systematics and Evolution of Heteroptera: 25 years of progress. Annual Review of Entomology, 56: 487-510. DOI: https://doi.org/10.1146/ annurev-ento-120709-144833

Wilcken, CF, EP Soliman, DALA, Nogueira, LRR Barbosa, TKRR Dias, FPJ Filho \& RRJ Oliveira, 2010. Bronze Bug Thaumastocoris peregrinus Carpintero \& Dellapé, (Heteroptera: Thaumastocoridae) on Eucalyptus in Brazil and its distribution. Journal of Plant Protection Research, 50: 201-205. DOI: https://doi.org/10.2478/v10045-0100034-0 


\section{Suggestion citation:}

Vélez, M, LA Coelho, DS Martins \& PSF Ferreira, 2020. Survey of the Heteroptera (Hemiptera) on pastures from Espírito Santo state, Brazil: new records, range extension, and notes of potential pests. EntomoBrasilis, 13: e907.

Available in: doi: 10.12741/ebrasilis.v13.e907

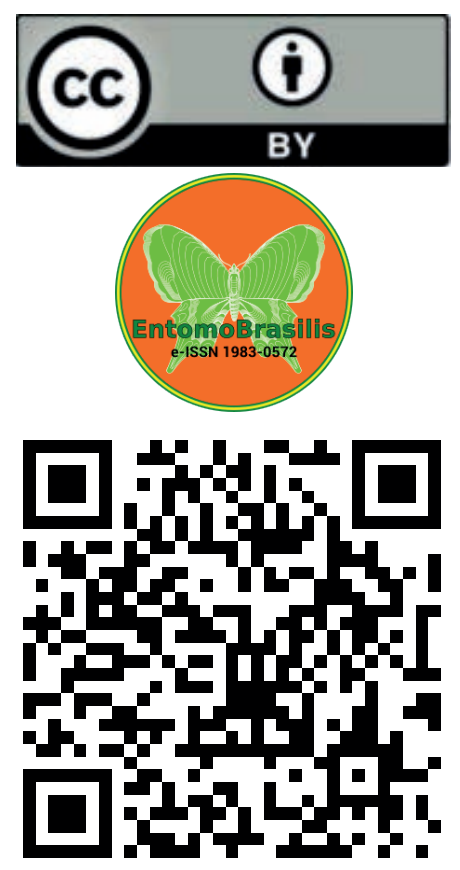

